\title{
CHARACTERIZATION OF THE INNER KNOT OF THE CRAB: THE SITE OF THE GAMMA-RAY FLARES?
}

\author{
Alexander Rudy ${ }^{1}$, Dieter Horns ${ }^{2}$, Andrea Deluca $^{3,4}$, JefFery Kolodziejczak $^{5}$, Allyn Tennant $^{5}$, Yajie Yuan ${ }^{6}$, \\ Rolf Buehler $^{7}$, Jonathon Arons ${ }^{8}$, Roger Blandford ${ }^{6}$, Patrizia Caraveo ${ }^{3,4}$, Enrico Costa ${ }^{9}$, Stephan Funk ${ }^{6}$, \\ Elizabeth Hays ${ }^{10}$, Andrei Lobanov ${ }^{11}$, Claire Max ${ }^{1}$, Michael Mayer ${ }^{7}$, Roberto Mignani ${ }^{3,12}$, Stephen L. O’Dell ${ }^{5}$, \\ Roger Romani $^{6}$, Marco Tavani $^{3,4}$, and Martin C. Weisskopf ${ }^{5}$ \\ ${ }^{1}$ Department of Astronomy \& Astrophysics, University of California, Santa Cruz, CA 95064, USA \\ ${ }^{2}$ Institut für Experimentalphysik, Universität Hamburg, Luruper Chaussee 149, D-22761 Hamburg, Germany \\ ${ }^{3}$ INAF-IASF Milano, via E. Bassini 15, I-20133 Milano, Italy \\ ${ }^{4}$ INFN Pavia, via A. Bassi 6, I-27100 Pavia, Italy \\ ${ }^{5}$ NASA Marshall Space Flight Center, Astrophysics Office (ZP12), Huntsville, AL 35812, USA \\ ${ }^{6}$ W. W. Hansen Experimental Physics Laboratory, Kavli Institute for Particle Astrophysics and Cosmology, Stanford University, Stanford, CA 94305, USA \\ ${ }^{7}$ DESY, Platanenallee 6, D-15738 Zeuthen, Germany \\ ${ }^{8}$ Astronomy Department and Theoretical Astrophysics Center, University of California, Berkeley, 601 Campbell Hall, Berkeley, CA 94720, USA \\ ${ }^{9}$ INFN Roma Tor Vergata, via della Ricerca Scientifica 1, I-00133 Roma, Italy \\ ${ }^{10}$ NASA Goddard Space Flight Center, Astrophysics Science Division, Greenbelt, MD 20771, USA \\ ${ }_{11}$ Max-Planck-Institut für Radioastronomie, auf dem Hügel 69, D-53121 Bonn, Germany \\ ${ }^{12}$ Kepler Institute of Astronomy, University of Zielona Gora, Lubuska 2, 65-265 Zielona Gora, Poland \\ Received 2015 April 17; accepted 2015 August 3; published 2015 September 16
}

\begin{abstract}
A particularly intriguing recent result from $\gamma$-ray astronomy missions is the detection of powerful flares from the Crab Nebula, which challenges the current understanding of pulsar wind nebulae and acceleration mechanisms. To search for the production site(s) of these flares, we conducted a multi-wavelength observing campaign using Keck, the Hubble Space Telescope (HST), and the Chandra X-ray Observatory. As the short timescales of the $\gamma$-ray flares ( $\lesssim 1$ day) suggest a small emitting region, the Crab's inner knot (about 0.6 arcsec from the pulsar) is a candidate site for such flaring. This paper describes observations of the inner knot, seeking to understand its nature and possible relationship with $\gamma$-ray flares. Using singular-value decomposition, analysis of the HST images yielded results consistent with traditional methods while substantially reducing some uncertainties. These analyses show that the knot's intrinsic properties (especially size and brightness) are correlated with its (projected) separation from the pulsar. This characterization of the inner knot helps in constraining standard shock model parameters, under the assumption that the knot lies near the shocked surface. While the standard shock model gives good agreement in several respects, two puzzles persist: (a) the observed angular size of the knot relative to the pulsar-knot separation is much smaller than expected; and (b) the variable high degree of polarization (reported by others) is difficult to reconcile with a highly relativistic downstream flow. However, the IR-optical flux of the inner knot is marginally consistent with the shock accelerating most of the Nebula's optical-emitting particles.
\end{abstract}

Key words: ISM: jets and outflows - ISM: supernova remnants - pulsars: individual (Crab) - techniques: image processing

\section{INTRODUCTION}

The Crab pulsar and its nebula are one of the most studied targets in the sky at all wavelengths. It serves as a test bed for pulsar theories as well as, more generally, for astrophysical non-thermal processes. A very thorough review of the Crab system, including high spatial resolution observations with Hubble Space Telescope (HST) and Chandra, showing the complex, dynamic interaction of the pulsar wind with the surrounding medium, has been compiled by Hester (2008). A more recent review by Bühler \& Blandford (2014) also includes the saga of the $\gamma$-ray flares.

A crucial question implied by the observations of the $\gamma$-ray flares is "how are the particles being accelerated to sustain the flaring behavior?" Radiating particles must have PeV energies to emit synchrotron radiation at the observed $\gamma$-ray wavelengths. A mechanism is needed that can accelerate particles to such high energies in less than one gyration time in the ambient magnetic field. For example, the large flare of 2011 April produced a radiant energy equivalent to the energy stored in the mean magnetic field within a region of order $2 \times 10^{14} \mathrm{~m}$ subtending an angle $\approx 0 ! 3$ at $2 \mathrm{kpc}$, consistent with the emission-region size estimated from light travel time arguments (Weisskopf et al. 2013).

An intense theoretical effort is underway to explain the variable Crab behaviors (e.g., Komissarov \& Lyutikov 2011; Uzdensky et al. 2011; Yuan et al. 2011; Arons 2012; Bykov et al. 2012; Cerutti et al. 2012, 2013; Clausen-Brown \& Lyutikov 2012; Lyubarsky 2012; Lyutikov et al. 2012; Sturrock \& Aschwanden 2012; Baty et al. 2013; Teraki \& Takahara 2013). However, the mechanism driving the flares, their impulsive nature, the $\approx 12$-month recurrence time, and the location remain unknown. Possible explanations include plasma instabilities in the nebula, magnetic reconnection, discontinuity in the pulsar wind acceleration, emission from the anvil, emission from the inner knot, or portions of the termination shock. See, for example, the review by Hester (2008) for the association of specific features with this nomenclature.

There are a number of reasons to suspect that the feature known as the "inner knot" should be considered as a possible site for the origin of the flares (e.g., Komissarov \& Lyutikov 2011). First, the luminosity of the flares accounts for a relatively high fraction of the pulsar spin-down luminosity 
(assuming a radiation efficiency of 3\%-5\%), suggesting an origin in a region close to the pulsar (Tavani et al. 2011). Second, the very short variability timescales combined with causality arguments, strongly constrain the size of the flare's emitting region (Striani et al. 2011; Buehler et al. 2012).

A multi-wavelength campaign to study the Crab, described in Weisskopf et al. (2013), is being performed. Another $\gamma$-ray flare - the second largest observed to date-was detected with Fermi/LAT in 2013 March (Mayer et al. 2013). This triggered further observations with Keck, with the $H S T$, and with the Chandra X-ray Observatory. Here we concentrate on what was learned about the inner knot as a consequence of this campaign. We note that very-high-energy $(E \geqslant 100 \mathrm{GeV})$ contemporaneous data from the HESS (H.E.S.S. Collaboration et al. 2014) and VERITAS (Aliu et al. 2014) Cherenkov-telescope arrays found no significant correlation of $\mathrm{TeV}$ flux with the flare observed with Fermi/LAT.

The observations are presented in Section 2. The measured properties of the knot are given in Section 3. Section 4 compares observations of the inner knot at the different wavelengths. A comparison and an examination of possible correlations of the knot's properties with $\gamma$ rays detected using the Fermi/LAT are discussed in Section 5. Section 6 discusses theoretical implications. Section 7 summarizes our findings.

\section{THE OBSERVATIONS}

The feature we refer to as the "inner knot" (aka "knot 1," or "synchrotron knot") was discovered by Hester et al. (1995) in a detailed study of the Crab using high-resolution images taken with $H S T$. The feature, although resolved in those data, is small $(\approx 0$ !" 1$)$ and close to the pulsar $(\approx 0 " .6)$. The feature was first suggested to be associated with a shock or instability in the jet $\approx 1500$ AU from the pulsar, due to its good alignment with the jet and elongation perpendicular to the jet. Later Komissarov \& Lyubarsky (2003) proposed, based on two-dimensional (2D) magnetohydrodynamic (MHD) simulations of the pulsar wind nebula, that the knot represents Doppler-boosted emission from an oblique termination shock. Synthetic synchrotron images from these 2D simulations (Komissarov \& Lyutikov 2011), and more recently three-dimensional (3D) simulations (Porth et al. 2014), indeed show a knot-like feature when the system is viewed from the proper (and reasonable) orientation. Moreover, the observed high degree of polarization and a position angle aligned with the symmetry axis (Moran et al. 2013) seems to lend further support to the oblique shock scenario.

The salient features of the inner knot deduced prior to our observations indicate that it has a power-law spectrum $F_{\nu} \propto \nu^{-\alpha}$ with index $\alpha \sim 0.8$ in the IR to optical (Sollerman 2003; Melatos et al. 2005) and, as most recently measured by Moran et al. (2013), the flux varies, the position appears to change, the degree of polarization is very large but does not appear to change with the flux (but see the discussion at the end of Section 5), and the magnetic field (determined from the position angle of the optical polarization) is orthogonal to the axis of symmetry (presumably the spin axis) and consistent with the direction of the field for the rest of the inner nebula.

\subsection{Keck}

We obtained $K^{\prime}$ - and $H$-band near-infrared (NIR) images of the inner $40^{\prime \prime}$ of the Crab Nebula using the Keck Near-infrared
Table 1

Summary of the 12 Keck Observations

\begin{tabular}{lcclc}
\hline \hline$\#$ & Date & MJD $^{\mathrm{a}}$ & Instrument $^{\mathrm{b}}$ & Exposure (s) \\
\hline K1 & 2012 Feb 08 & 55965.285 & NIRC2-wide & 420 \\
K2 & 2012 Mar 05 & 55991.319 & NIRC2-wide & 420 \\
K3 & 2012 Dec 22 & 56283.408 & NIRC2-wide & 1250 \\
K4 & 2012 Dec 23 & 56284.410 & NIRC2-narrow & 870 \\
K5 & 2012 Dec 24 & 56285.388 & NIRC2-narrow & 1350 \\
K6 & 2012 Dec 25 & 56286.390 & NIRC2-wide & 1125 \\
K7 & 2013 Feb 06 & 56329.308 & NIRC2-narrow & 2020 \\
K8 & 2013 Oct 22 & 56587.448 & NIRC2-narrow & 640 \\
K9 & 2014 Jan 09 & 56666.274 & NIRC2-wide & 1560 \\
K10 & 2014 Jan 09 & 56666.377 & NIRC2-narrow & 2580 \\
K11 & 2014 Jan 17 & 56674.325 & NIRC2-wide & 2270 \\
K12 & 2014 Jan 17 & 56674.394 & NIRC2-narrow & 1260 \\
\hline
\end{tabular}

Notes.

${ }^{\text {a }}$ Quoted MJD is at the midpoint of the observation.

b Plate scale is 0 ". 04/pixel for NIRC2-wide; 0." 01/pixel for NIRC2-narrow.

Camera 2 (NIRC2; K. Matthews, PI), with laser guide star adaptive optics (AO; Wizinowich et al. 2006). From 2012 February to 2014 January, we obtained 11 observations of the nebula during $\gamma$-ray quiescent periods and 1 target-ofopportunity observation (K8) triggered by $\gamma$-ray flares. Table 1 summarizes the Keck observations.

Data reduction was carried out using the pipeline developed by Ghez et al. (2008). Individual frames were sky-subtracted, flat-fielded, bad-pixel-corrected, and corrected for the NIRC2's camera distortion. We used the solution developed by Yelda et al. (2010), which matched the positions of stars in the globular cluster M92 measured with HST to those measured in the NIRC2. Frames were checked to ensure that they showed no irregularities in the point-spread function (PSF), and that the Strehl ratio was at least $\sim 0.10$. Individual frames were then combined using the Drizzle algorithm (Fruchter \& Hook 2002) to provide sub-pixel accuracy and high signal-to-noise images for analysis and modeling (Section 3.1 and Appendix).

The observations were conducted with two different pixel scales, using the NIRC2 "wide" camera, with 0". 04 pixels and a $40^{\prime \prime} \times 40^{\prime \prime}$ field of view, and the NIRC2 "narrow" camera, with 0 ". 01 pixels and a $10^{\prime \prime} \times 10^{\prime \prime}$ field of view. The total effective exposure times ranged from 420 to $\approx 2600 \mathrm{~s}$. The shorter exposures result in optimal use of 1-hr Target-of-opportunity interrupts-including acquisition, calibration, and sky measurements.

The spatial resolution of Keck's NIRC2 with AO proved to be very effective for resolving the detailed structure and position of the knot. Figure 1 shows the 12 NIRC2 images. In the narrow camera, the center of the pulsar and the center of the knot are separated by $\approx 60$ pixels. Typical values for the signalto-noise ratio of the knot are 5. The PSF of NIRC2 with AO varies slightly in time due to the variable performance of the AO system. As the pulsar and knot are well-resolved from each other in the Keck images, we chose not to deconvolve the PSF from the Keck images.

\section{2. $H S T$}

Since 2012 January, we have obtained 22 observations using the HST Advanced Camera for Surveys (ACS) Wide-field Channel (WFC), with 0". 05 pixels. Each observation comprises four exposures totaling $2000 \mathrm{~s}$ and uses a standard 4-point 



K9 2014-01-09 Narrow

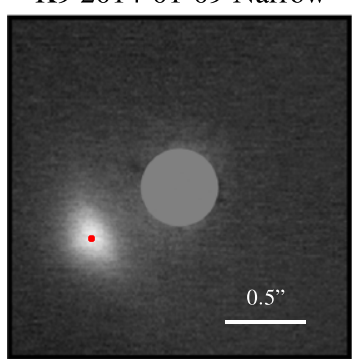

K2 2012-03-05 Wide

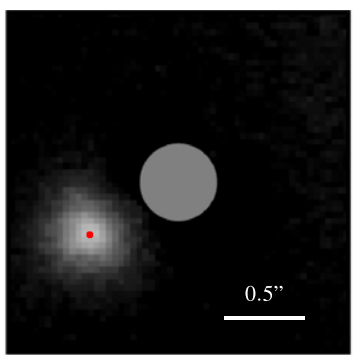

K6 2012-12-25 Wide

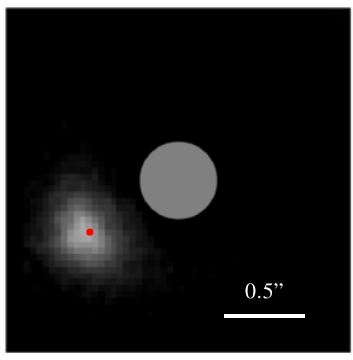

K10 2014-01-09 Wide

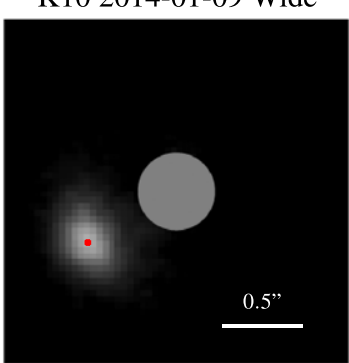

K3 2012-12-22 Wide

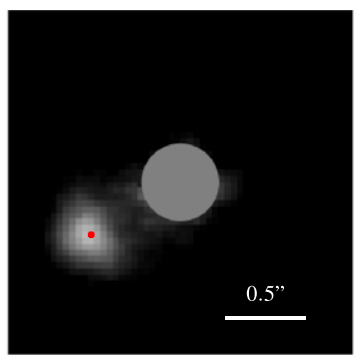

K7 2013-02-06 Narrow

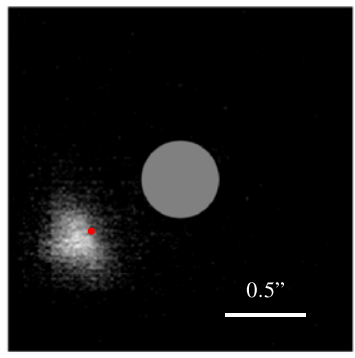

K11 2014-01-17 Narrow

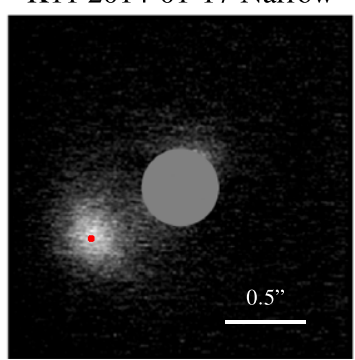

K4 2012-12-23 Narrow

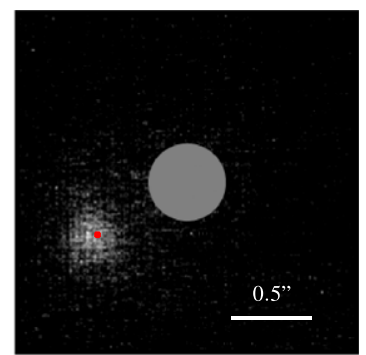

K8 2013-10-22 Narrow

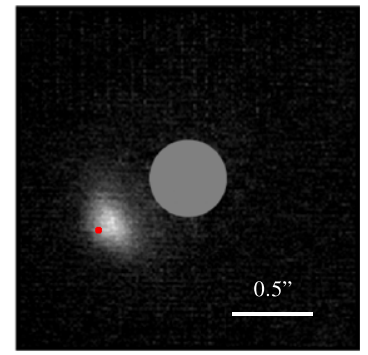

K12 2014-01-17 Wide

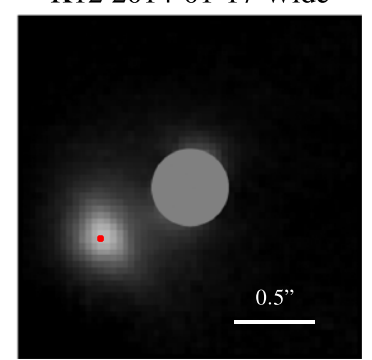

Figure 1. Individual images of the inner knot, with the pulsar removed, from Keck NIRC2 with laser guide star adaptive optics (AO). In each image, the PSF of the pulsar has been subtracted using the scaled PSF of a nearby star and replaced with a gray circle at the original location of the pulsar. Each image is 2 !! 2 by 2 !. 2 . The images are from the NIRC2-wide camera $\left(0{ }^{\prime \prime} 04\right.$ pixels) or the NIRC2-narrow camera $\left(0{ }^{\prime \prime} 01\right.$ pixels). All images were taken using the $K^{\prime}$ filter. For positional reference, the red dot marks the average position of the inner knot across all 12 observations.

BOX dithering pattern to fill the inter-chip gap as well as to allow efficient cosmic ray cleaning. We employed the F550M filter, which is well-suited to sampling continuum emission from the Crab with almost no contamination from line emission.

Table 2 summarizes the observations, listing the date and the mean observing time. The Appendix discusses in detail our analyses of the $22 \mathrm{HST} / \mathrm{ACS}$ images. Figure 2 displays the processed images, in which we have removed the effects of the HST/ACS WFC PSF using singular-value decomposition (SVD) and Richardson-Lucy deconvolution.

\subsection{Chandra}

Our approach for searching for X-ray emission from the inner knot with Chandra involved a special use of both the high time resolution and high spatial resolution of the Highresolution Camera for Spectroscopy (HRC-S) array in timing mode. This approach combines the best spatial resolution and the best time resolution afforded by the Chandra instrumentation. To achieve the time resolution, it is vital that the counting rate be below the rate where telemetry is saturated (see, e.g., Tennant et al. 2001). Consequently, the Low-energy Transmission Grating was inserted into the optical path to reduce the flux. As inserting the grating is of itself insufficient to avoid telemetry saturation, the trigger threshold of the HRC-S was raised to reduce the rate of detected events. This approach was first successfully used in a trial experiment (ObsID 11245) on 2010 November 16.

The next set of observations in this mode was initiated by us on 2013 March 5 as part of a sequence triggered by a $\gamma$-ray flare (Ojha et al. 2013). Unfortunately, the gain of the HRC had dropped since 2010. Consequently, the trigger threshold was too high during our first observation on March 5-6, reducing the counting rate from the pulsar to about a quarter of that expected, dramatically decreasing the number of detected counts. The next HRC observation was to take place on March 10 and the observation had already been fully planned with the commands to execute on board the spacecraft. To replan at this stage would require an extremely short turnaround, yet it was accomplished. Finally, beginning on 2013 October 22, we triggered another sequence of HRC observations in response to the announcement of another $\gamma$-ray flare (Buson et al. 2013).

We also consider ObsID 9765, taken on 2008 January 22. For this observation the HRC was operated in a more standard configuration with the threshold set at the nominal value. However, to reduce the count rate, a blade was inserted into the optical path, which is no longer allowed. This blade also reduced the diffracted flux in one direction. By chance, this reduction is greatest near the nominal location of the knot. This reduction, together with a long integration time for this observation, results in ObsID 9765 providing the best data 
Table 2

Summary of the 22 HST Observations

\begin{tabular}{|c|c|c|}
\hline$\#$ & Date & $\mathrm{MJD}^{\mathrm{a}, \mathrm{b}}$ \\
\hline H1 & 2012 Jan 08 & 55934.797 \\
\hline $\mathrm{H} 2$ & 2012 Feb 10 & 55967.170 \\
\hline $\mathrm{H} 3$ & 2012 Mar 12 & 55998.414 \\
\hline $\mathrm{H} 4$ & 2012 Apr 22 & 56039.850 \\
\hline H5 & 2012 Aug 16 & 56155.336 \\
\hline H6 & 2012 Sep 10 & 56180.960 \\
\hline $\mathrm{H} 7$ & 2013 Jan 10 & 56302.992 \\
\hline H8 & 2013 Feb 24 & 56347.055 \\
\hline $\mathrm{H} 9$ & 2013 Mar 06 & 56357.023 \\
\hline H10 & 2013 Apr 01 & 56383.320 \\
\hline H11 & 2013 Apr 14 & 56396.113 \\
\hline H12 & 2013 Aug 13 & 56517.710 \\
\hline H13 & 2013 Oct 20 & 56585.793 \\
\hline H14 & 2013 Oct 29 & 56594.700 \\
\hline H15 & 2013 Dec 01 & 56627.273 \\
\hline H16 & 2014 Jan 20 & 56677.530 \\
\hline H17 & 2014 Apr 13 & 56760.900 \\
\hline H18 & 2014 Aug 10 & 56879.375 \\
\hline H19 & 2014 Aug 21 & 56890.094 \\
\hline $\mathrm{H} 20$ & 2014 Nov 16 & 56977.820 \\
\hline $\mathrm{H} 21$ & 2015 Jan 21 & 57043.620 \\
\hline $\mathrm{H} 22$ & 2015 Apr 16 & 57128.670 \\
\hline
\end{tabular}

Notes.

${ }^{\text {a }}$ Quoted MJD is at the midpoint of the observation.

b Plate scale is 0 ." $05 /$ pixels.

for study of the knot. Table 3 summarizes the relevant Chandra observations.

\section{PROPERTIES OF THE KNOT}

Each Keck (Figure 1) or HST (Figure 2) image obtained in this campaign clearly shows the inner knot at nominally 0 ". 65 southeast of the pulsar. As already noted in previous HST observations (Hester et al. 1995; Hester 2008; Moran et al. 2013), this feature is persistent but not static.

Here we report on our analysis of the Keck (Section 3.1) and HST (Section 3.2) images to measure the properties of the inner knot, changes with time, and correlations of intrinsic properties with the pulsar-knot separation. Figure 3 defines the lowestorder geometric properties of the knot: $r_{0}$, the projected separation of the knot's center from the pulsar; $\psi_{0}$, the position angle (east of north) on the sky from the pulsar to the knot's center; $\mathrm{FWHM}_{r}$ and $\mathrm{FWHM}_{t}$, the full widths at half maximum of the knot in the radial and tangential directions, respectively.

\subsection{Keck}

Table 4 lists the best-fit values of the model parameters for the inner knot for each of the 12 Keck observations based upon the analysis and modeling described in the Appendix. Besides listing the geometric properties $\left(r_{0}, \psi_{0}, \mathrm{FWHM}_{r}\right.$, and $\left.\mathrm{FWHM}_{t}\right)$, the table lists $F_{\mathrm{k}} / F_{\mathrm{p}}$, the ratio of the flux in the knot to that in the pulsar. We tabulate the flux ratio rather than an absolute flux because the measured fluxes of five stars in the NIRC2wide camera's field differed from one observation to the next. This occurs because the nebular background varies spatially across the detector in a non-trivial way, making it difficult to precisely determine the flux of any given source. Figure 4 plots $\psi_{0}, \mathrm{FWHM}_{r}$, and $\mathrm{FWHM}_{t}$, and $F_{\mathrm{k}} / F_{\mathrm{p}}$ against $r_{0}$ for the Keck data.

We test for a possible correlation of each property $p$ with separation $r_{0}$ from the pulsar, of the form $p\left(r_{0}\right)=$ $p\left(r_{1}\right)\left[r_{0} / r_{1}\right]^{q}$, where reference separation $r_{1}$ is the geometric average of the 12 Keck measurements. We fit the position angle of the knot, $\psi_{0}$, to the linear regression $\psi_{0}=\psi_{0}\left(\left\langle r_{0}\right\rangle\right)+$ $\psi_{0}^{\prime}\left(\left\langle r_{0}\right\rangle\right)\left[r_{0}-\left\langle r_{0}\right\rangle\right]$, where $\left\langle r_{0}\right\rangle$ is the arithmetic average. Table 5 tabulates the results of the regression analyses of the inner knot's intrinsic properties with its (projected) separation from the pulsar. Based upon the F-test probability, the powerlaw regression analysis finds marginally significant correlations of the radial width, tangential width, and peak intensity (surface brightness) with separation. The correlations to the separation of the knot's flux and position angle are not significant.

\subsection{HST}

Table 6 lists the best-fit values of the model parameters for the inner knot for each of the 22 HST observations based upon the modeling and analysis described in the Appendix. Besides listing the geometric properties $\left(r_{0}, \psi_{0}, \mathrm{FWHM}_{r}\right.$, and $\left.\mathrm{FWHM}_{t}\right)$, the table lists $F_{\mathrm{k}} / F_{\mathrm{p}}$, the ratio of the flux in the knot to that in the pulsar, and $S_{\mathrm{k}} / F_{\mathrm{p}}$, the peak surface brightness scaled to the pulsar flux. Although we expected the pulsar flux to be steady, we measured small $(2.5-\sigma)$ variations and thus decided to work with the flux ratio $F_{\mathrm{k}} / F_{\mathrm{p}}$.

In the Appendix we discuss two ways of analyzing the HST data. Table 6 presents the results for one of these methods. However, our general conclusions concerning the knot's optical properties are for the most part the same for either analysis. Figure 5 plots the knot properties versus the pulsarknot separation, based upon the HST data.

As with the Keck data, we perform a regression analysis of each property $p$ with separation $r_{0}$. (Table 7). Similarly, $r_{1}$ is the geometric average of the $22 H S T$ observations; $\left\langle r_{0}\right\rangle$, the arithmetic average.

Based upon the F-test probability, the power-law regression analysis finds statistically significant correlations of the tangential width $\mathrm{FWHM}_{t}$ with separation $r_{0}$ from the pulsar, as well as the previously known (Moran et al. 2013) anticorrelations of flux $F_{\mathrm{k}}$ and of peak surface brightness $S_{\mathrm{k}}$ with separation. The correlations of radial width $\mathrm{FWHM}_{r}$ and of position angle $\psi_{0}$ with separation $r_{0}$ are less strong. We note that we find similar correlations-except for the flux-using the more traditional HST analysis methods described in the Appendix.

\subsection{Chandra}

For the Chandra data, the main question is whether X-ray emission from the knot can be detected-especially in the vicinity of the bright, X-ray-emitting pulsar. The answer, unfortunately, is "No" and here we set an informative upper limit to the X-ray flux of the inner knot relative to that of the pulsar.

Table 8 shows the number of counts in each of three extraction regions: (1) centered on the approximate location of the optical knot, (2) centered on the pulsar, and (3) placed on a reference region to estimate the background. Details may be found in the Appendix and are illustrated in Figure 6. The analysis was performed both for the full phase-averaged data and for the data at pulse minimum. Consider, for example, the 


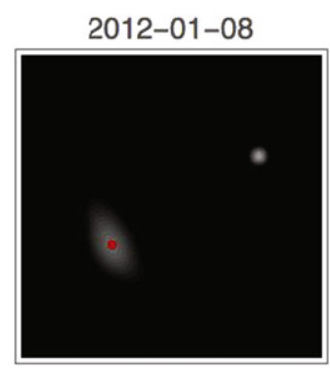

2012-08-16

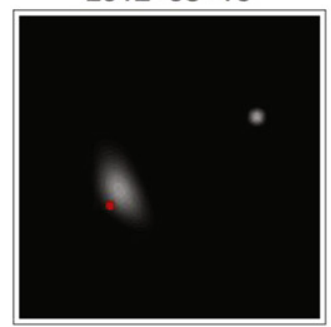

2013-03-06

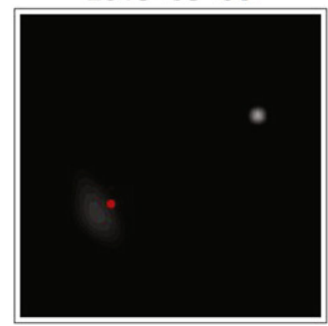

2013-10-20

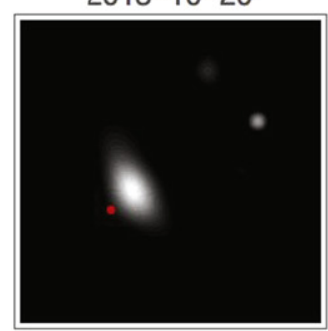

2014-04-13

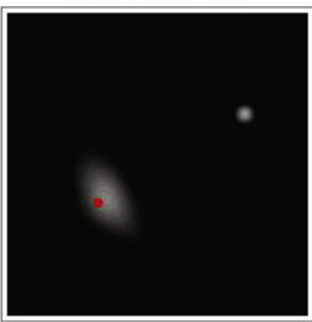

2012-02-10

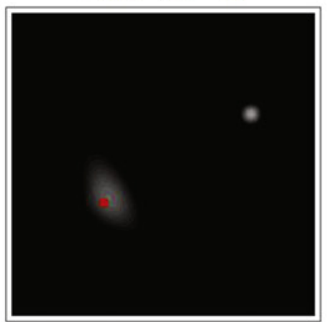

2012-09-10

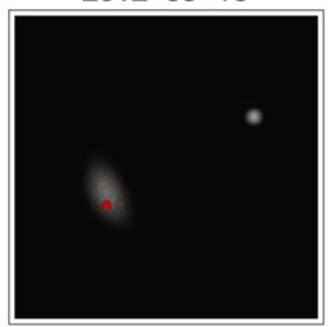

2013-04-01

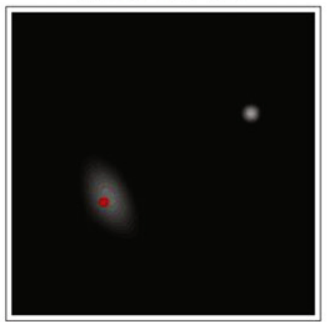

2013-10-29

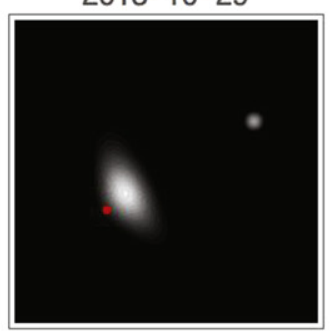

2014-08-10

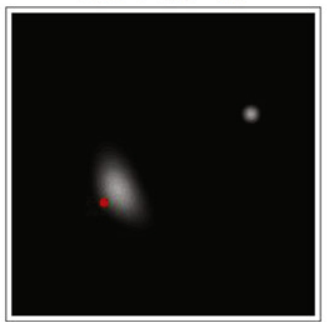

2015-01-21

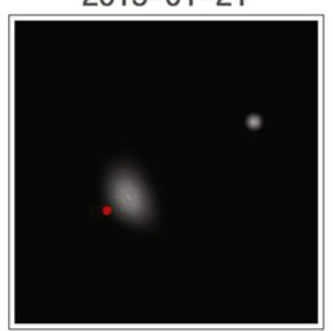

2012-03-12

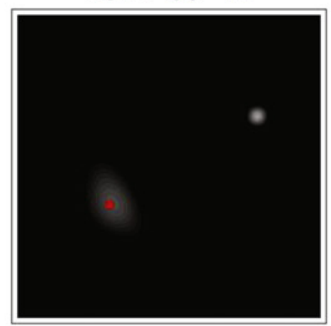

2013-01-10

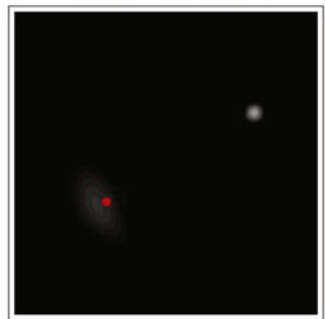

2013-04-14

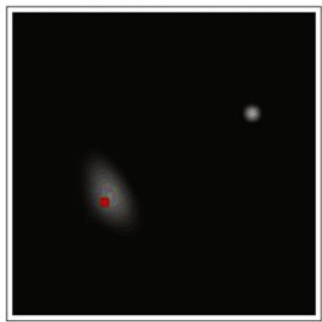

2013-12-01

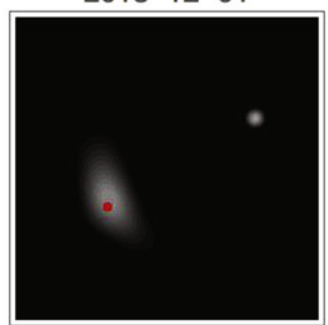

2014-08-21

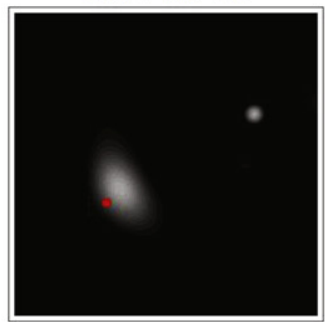

2015-04-16

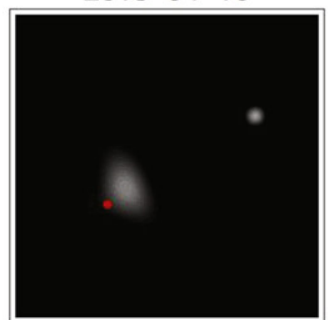

Figure 2. HST-SVD-processed images of the Crab pulsar and inner knot after removing the effects of the HST/ACS WFC point-spread function. For positional reference, the red dot in each image marks the position of the inner knot during the first of these 22 observations. The HST observations of 2013 March 06 (H9) and of 2013 October 29 (H14) each occurred at the peak of a $\gamma$-ray flare (Figure 8). 
Table 3

Summary of the Chandra Observations

\begin{tabular}{lcccc}
\hline \hline$\#$ & ObsID & Date & MJD $^{\mathrm{a}}$ & Exposure (s) \\
\hline C1 & 09765 & 2008 Jan 22 & 54487.670 & 95082 \\
C2 & 11245 & 2010 Nov 16 & 55516.294 & 22039 \\
C3 & 14684 & 2013 Mar 05 & 56356.972 & 19485 \\
C4 & 14686 & 2013 Mar 10 & 56361.338 & 20052 \\
C5 & 14687 & 2013 Mar 17 & 56368.324 & 16718 \\
C6 & 16244 & 2013 Oct 19 & 56584.658 & 20048 \\
C7 & 16246 & 2013 Oct 22 & 56587.515 & 20013 \\
C8 & 16247 & 2013 Oct 24 & 56589.007 & 19770 \\
\hline
\end{tabular}

Note.

a Quoted MJD is at the start of the observation.

phase-averaged data for ObsID 11245. Comparing the inner knot counts to the background (Table 8) we find an excess of 167 counts. If the excess were really due to the knot, we would accordingly expect an excess of $50(=167 \times 0.3)$ counts at phase minimum. As this was not the case, we attribute the excess to the wings of the PSF from the pulsar and posit that it is due to the spring/fall asymmetry discussed in the Appendix. As the impact of that asymmetry is below the statistical noise for the data taken at phase minimum, we then use the phase minimum data to derive upper limits. We convert our phase minimum upper limit into a phase-averaged upper limit by accounting for the 0.3 phase duration of the minimum and then divide by the phase average pulsar flux. The results are listed in the last column in Table 8 .

\section{COMPARISON AMONG OBSERVATIONS OF THE INNER KNOT}

Our Chandra observations were unable to detect X-ray flux from the knot, setting a $3 \sigma$ upper limit to the ratio of knot flux to pulsar flux $F_{\mathrm{k}} / F_{\mathrm{p}}<0.0022$. This upper limit is $20-40$ times smaller than the ratio measured in the optical $\left(F_{\mathrm{k}} / F_{\mathrm{p}}=\right.$ $0.044 \pm 0.001)$ and in the near-IR $\left(F_{\mathrm{k}} / F_{\mathrm{p}}=0.063 \pm 0.003\right)$, indicating that the optical-X-ray spectral index of the knot is significantly steeper (by at least 0.5 ) than that of the pulsar.

Both our infrared and optical observations detected time variability in the pulsar-knot separation, with roughly consistent separations when measured contemporaneously (see the lower portion of Figure 7). Correlations of knot properties with pulsar-knot separation are very strong for the optical measurements but rather weak for the infrared measurements. We attribute this difference to a combination of factors-including poorer statistics for the much shorter NIR measurements and a number of systematic effects, resulting in part from the NIR PSF and background varying across the field and in time. As the lower portion of Figure 7 and Table 4 indicate, NIR-measured separations at essentially the same epoch exhibit a scatter that is substantially larger than the quoted statistical errors in the measurement, thus suggesting the presence of unaccounted systematic errors.

\section{COMPARISON WITH $\gamma$-RAY FLUXES}

It is clear from the previous sections that the intrinsic properties of the optical knot are correlated with its projected separation from the pulsar. The correlation for the NIR knot is but marginally significant. Thus, in searching for any relationship between the knot and the $\gamma$-ray flux, we concentrate on the

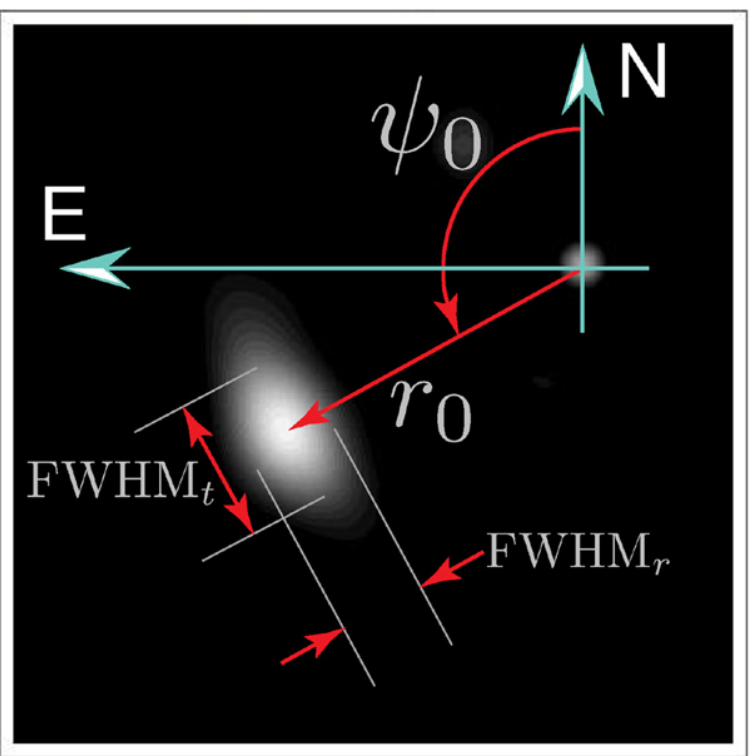

Figure 3. Geometric properties of the inner knot: $r_{0}$, the projected separation of the knot from the pulsar; $\psi_{0}$, the position angle (east of north) on the sky of the pulsar-knot separation; $\mathrm{FWHM}_{r}$, the full width at half maximum of the knot in the radial direction; and $\mathrm{FWHM}_{t}$, the FWHM of the knot in the tangential direction. The underlying image is from the 2013 October 20 HST observation (H13), which exhibits the minimum separation and maximum optical flux observed during this campaign.

pulsar-knot separation versus the $\gamma$-ray flux. The analysis performed to reduce the Fermi/LAT data is the same as that described in Mayer et al. (2013).

Table 9 lists the dates and separations measured in the Keck and in the HST observations, followed by the corresponding 12-hr-average Fermi/LAT $\geqslant 100 \mathrm{MeV}$ fluxes. The upper portion of Figure 7 shows the Fermi/LAT flux as a function of time covering the interval when our Keck and HST observations were made. The vertical lines identify times when observations were made with Keck (red) or with HST (green). The lower portion of Figure 7 plots the pulsar-knot separations on the same timescale. Figure 8 expands Figure 7 at the times of the three largest $\gamma$-ray flaring episodes during our observational campaign. Note that the HST observations of 2013 March 06 (H9) and of 2013 October 29 (H14) each fortuitously occurred within the peak of a $\gamma$-ray flare.

Interestingly, a comparison of the $\gamma$-ray light curve with the time series of pulsar-knot separations (Figure 7) finds that the most energetic $\gamma$-ray flare (2013 March) during the observational campaign occurred near the time of the greatest pulsarknot separation. Curiously, however, this comparison also finds that the second most energetic flaring episode (2013 October) occurred near the time of the least pulsar-knot separation. The third most energetic flaring episode (2014 August) took place while the separation was midway between the average and minimum measured during the campaign. The inner knot's excursion near the 2013 March $\gamma$-ray flare and its incursion near the 2013 October flare were transient, with timescales of a few weeks-significantly longer than the time scale of a few days (or less) of a $\gamma$-ray flare. The lesser incursion of the knot near the 2014 August flaring episode was even slower.

As the timescales for motion of the inner knot appear to be an order of magnitude longer than those for the $\gamma$-ray flaring, there can be no simple correlation of $\gamma$-ray flux with pulsar- 
Table 4

Knot Properties Based upon Analysis of the Keck Data

\begin{tabular}{|c|c|c|c|c|c|}
\hline \# & $\begin{array}{l}r_{0} \\
\prime \prime\end{array}$ & $\underset{\circ}{\psi_{0}}$ & $\underset{\prime \prime}{\mathrm{FWHM}_{r}}$ & $\underset{\prime \prime}{\mathrm{FWHM}_{t}}$ & $\begin{array}{l}F_{\mathrm{k}} \\
F_{\mathrm{p}} \\
\end{array}$ \\
\hline K1 & $0.6711 \pm 0.0015$ & $118.50 \pm 0.16$ & $0.3931 \pm 0.0040$ & $0.4719 \pm 0.0051$ & $0.0639 \pm 0.0045$ \\
\hline K2 & $0.6577 \pm 0.0015$ & $121.00 \pm 0.13$ & $0.4121 \pm 0.0035$ & $0.4252 \pm 0.0042$ & $0.0626 \pm 0.0067$ \\
\hline K3 & $0.6893 \pm 0.0011$ & $122.64 \pm 0.11$ & $0.3215 \pm 0.0028$ & $0.3948 \pm 0.0039$ & $0.0804 \pm 0.0074$ \\
\hline K4 & $0.7118 \pm 0.0021$ & $120.96 \pm 0.18$ & $0.3401 \pm 0.0051$ & $0.4081 \pm 0.0059$ & $0.0473 \pm 0.0003$ \\
\hline K5 & $0.6981 \pm 0.0007$ & $121.20 \pm 0.07$ & $0.2983 \pm 0.0019$ & $0.3767 \pm 0.0024$ & $0.0555 \pm 0.0005$ \\
\hline K6 & $0.7164 \pm 0.0010$ & $120.79 \pm 0.12$ & $0.3375 \pm 0.0029$ & $0.4238 \pm 0.0042$ & $0.0599 \pm 0.0085$ \\
\hline K7 & $0.7756 \pm 0.0013$ & $121.93 \pm 0.12$ & $0.3355 \pm 0.0034$ & $0.4049 \pm 0.0038$ & $0.0662 \pm 0.0004$ \\
\hline K8 & $0.5722 \pm 0.0004$ & $120.33 \pm 0.06$ & $0.2216 \pm 0.0011$ & $0.3172 \pm 0.0015$ & $0.0621 \pm 0.0005$ \\
\hline K9 & $0.6543 \pm 0.0006$ & $121.46 \pm 0.08$ & $0.2958 \pm 0.0018$ & $0.4030 \pm 0.0024$ & $0.0708 \pm 0.0047$ \\
\hline K10 & $0.6257 \pm 0.0003$ & $115.77 \pm 0.03$ & $0.2689 \pm 0.0007$ & $0.3683 \pm 0.0009$ & $0.0663 \pm 0.0006$ \\
\hline K11 & $0.6371 \pm 0.0008$ & $121.49 \pm 0.10$ & $0.3119 \pm 0.0022$ & $0.3787 \pm 0.0029$ & $0.0647 \pm 0.0078$ \\
\hline K12 & $0.6438 \pm 0.0009$ & $118.99 \pm 0.09$ & $0.3248 \pm 0.0023$ & $0.3766 \pm 0.0025$ & $0.0574 \pm 0.0001$ \\
\hline
\end{tabular}
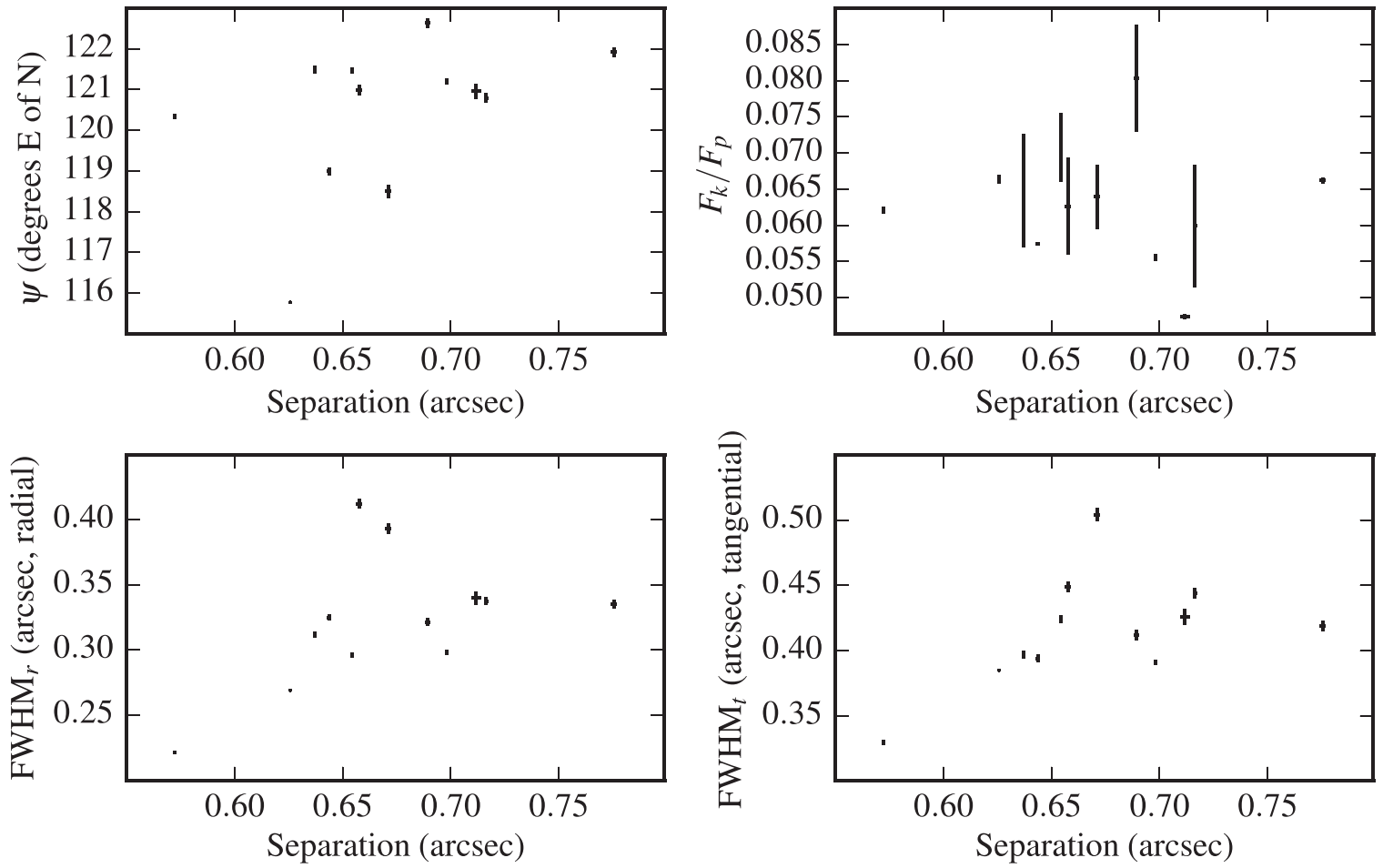

Figure 4. Variation of the Keck-measured knot properties vs. separation of the inner knot from the pulsar.

Table 5

Results of Regression Analyses for the 12 Keck Observations

\begin{tabular}{|c|c|c|c|c|}
\hline $\begin{array}{l}\text { Power-law for } r_{1}=00^{\prime \prime} 6692 \\
\text { Property }\end{array}$ & Unit & $p\left(r_{1}\right)$ & Power-law Index $q$ & Probability \\
\hline FWHM $_{r}$ & $" 1$ & $0.3180 \pm 0.0132$ & $1.19 \pm 0.54$ & $5.4 \mathrm{E}-2$ \\
\hline $\mathrm{FWHM}_{t}$ & $\prime \prime$ & $0.3941 \pm 0.0093$ & $0.75 \pm 0.31$ & $3.8 \mathrm{E}-2$ \\
\hline$S_{\mathrm{k}}$ & $F_{\mathrm{p}} /\left({ }^{\prime \prime}\right)^{2}$ & $0.439 \pm 0.033$ & $-2.14 \pm 0.97$ & $5.2 \mathrm{E}-2$ \\
\hline$F_{\mathrm{k}}$ & $F_{\mathrm{p}}$ & $0.0626 \pm 0.0025$ & $-0.20 \pm 0.53$ & 7.1E-1 \\
\hline \multicolumn{5}{|l|}{ Linear for $\left\langle r_{0}\right\rangle=0{ }^{\prime \prime} 6711$} \\
\hline Property & & $\psi_{0}\left(\left\langle r_{0}\right\rangle\right)$ & $d \psi_{0} / d r_{0}$ & \\
\hline$\psi_{0}$ & $\circ, \circ / 1$ & $120.42 \pm 0.51$ & $15.2 \pm 10.2$ & $1.7 \mathrm{E}-1$ \\
\hline
\end{tabular}


Table 6

Knot Properties Based upon SVD Analysis of the 22 HST Observations (See the Appendix)

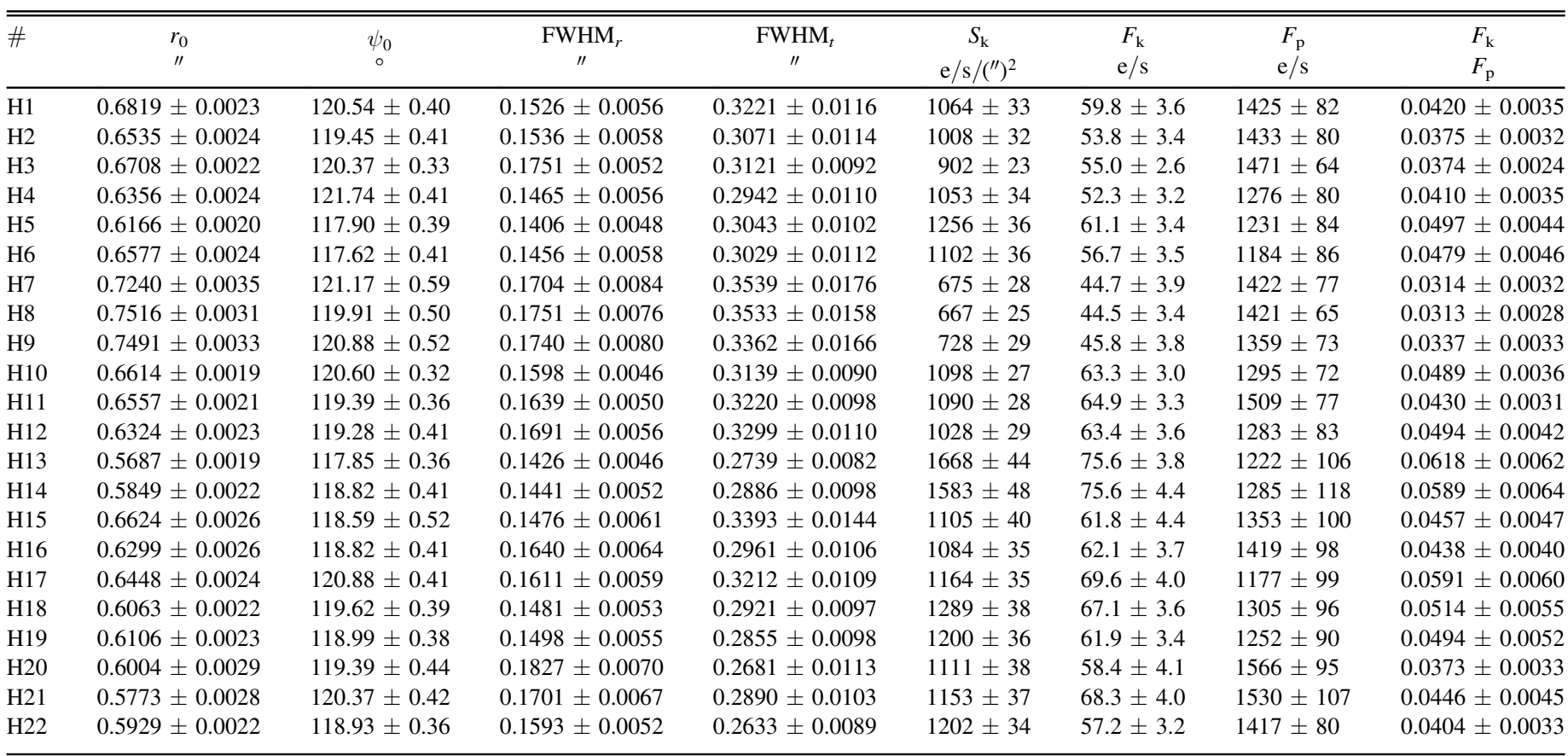
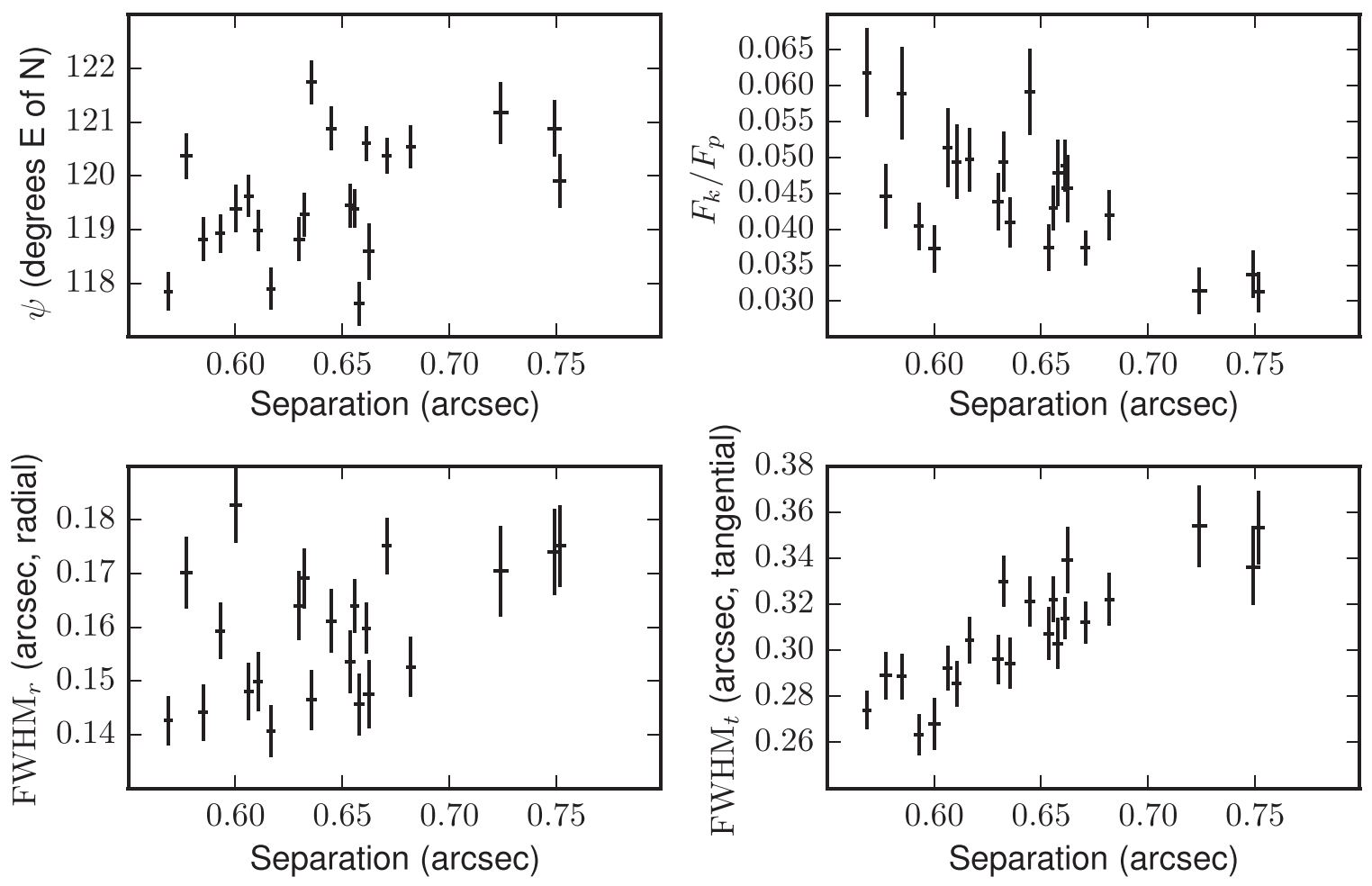

Figure 5. Variation of the HST-SVD-measured properties with separation of the inner knot from the pulsar.

knot separation. Indeed, a plot of $\gamma$-ray flux measurements that is coincident with the 22 HST and 12 Keck observations against the measured pulsar-knot separations (Figure 9) shows no significant correlation. The absence of a simple correlation, the relative sparsity of the near-IR and optical data, and the lack of a detailed physical model make it difficult to relate $\gamma$-ray flaring to changes in the pulsar-knot separation. Hence, while this behavior is intriguing, we have not succeeded in deducing a pattern relating the $\gamma$-ray flares to the pulsar-knot separation. Of course, it is also possible that no such relationship exists.

We note that Moran et al. (2013) used polarization observations from the HST archive to measure a possible change in the linear polarization of the inner knot that occurred a few weeks after an incursion of the inner knot in 2005 
Table 7

Results of Regression Analyses for the 22 HST Observations Based on the Data in Table 6

\begin{tabular}{|c|c|c|c|c|}
\hline $\begin{array}{l}\text { Power-law for } r_{1}=0{ }^{\prime \prime} 6421 \\
\text { Property }\end{array}$ & Unit & $p\left(r_{1}\right)$ & Power-law Index $q$ & Probability \\
\hline $\mathrm{FWHM}_{r}$ & " & $0.1584 \pm 0.0025$ & $0.41 \pm 0.21$ & $5.9 \mathrm{E}-2$ \\
\hline $\mathrm{FWHM}_{t}$ & " & $0.3067 \pm 0.0029$ & $0.92 \pm 0.12$ & $2.9 \mathrm{E}-7$ \\
\hline$S_{\mathrm{k}}$ & $F_{\mathrm{p}} /\left({ }^{\prime \prime}\right)^{2}$ & $0.796 \pm 0.029$ & $-2.77 \pm 0.47$ & $9.8 \mathrm{E}-6$ \\
\hline$F_{\mathrm{k}}$ & $F_{\mathrm{p}}$ & $0.0440 \pm 0.0014$ & $-1.70 \pm 0.40$ & 4.1E-4 \\
\hline \multicolumn{5}{|l|}{ Linear for $\left\langle r_{0}\right\rangle=0{ }^{\prime \prime} 6440$} \\
\hline Property & & $\psi_{0}\left(\left\langle r_{0}\right\rangle\right)$ & $d \psi_{0} / d r_{0}$ & \\
\hline$\psi_{0}$ & $\circ, \circ / 1$ & $119.60 \pm 0.22$ & $10.2 \pm 4.4$ & $3.2 \mathrm{E}-2$ \\
\hline
\end{tabular}

Table 8

Results of the Chandra Analysis

\begin{tabular}{|c|c|c|c|c|c|c|c|}
\hline \multirow[t]{2}{*}{ ObsID } & \multicolumn{3}{|c|}{ Pulse Average } & \multicolumn{3}{|c|}{ Pulse Minimum } & \multirow{2}{*}{$\begin{array}{c}F_{\mathrm{k}} / F_{\mathrm{p}} \\
3 \sigma\end{array}$} \\
\hline & Pulsar & Knot & Background & Pulsar & Knot & Background & \\
\hline 09765 & 51844 & 2963 & 3704 & 845 & 57 & 72 & $<0.0022$ \\
\hline Last 6 & 13549 & 2622 & 2503 & 203 & 39 & 44 & $<0.0067$ \\
\hline
\end{tabular}

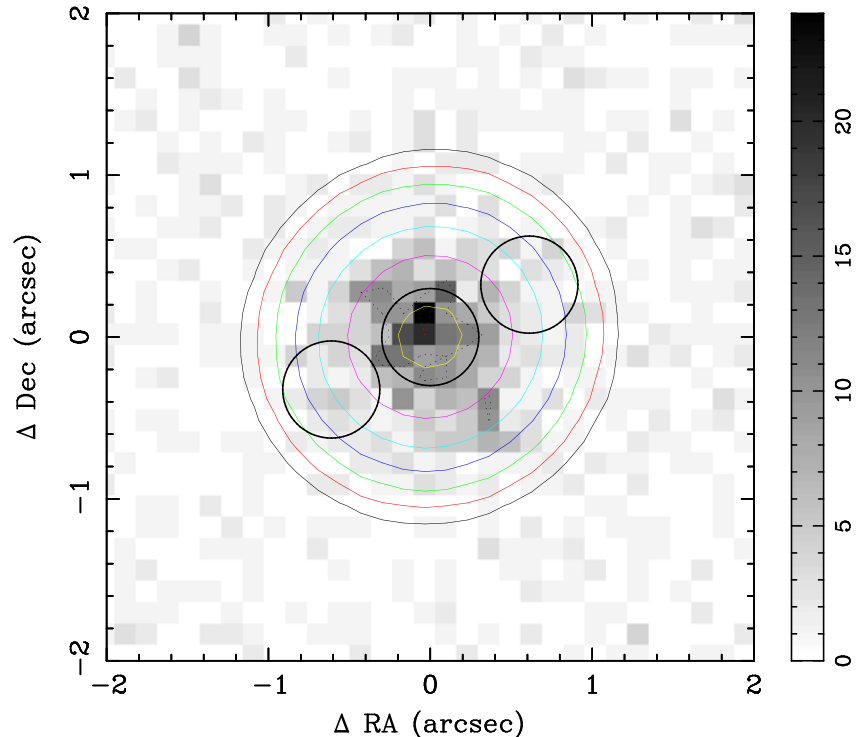

Figure 6. Chandra image at pulse minimum from the sum of the last six flaring data sets in Table 3. The three small black circles show 0 ". 3-radius extraction regions, the central of which is used to estimate the pulsar flux. The circle, at a position angle $\psi=120^{\circ}$ east of north and 0 " 65 from the pulsar, is roughly centered on the average optical location of the knot. Data in the opposite small circle (the one to the NW where N is up) are used to estimate background. Large circles show the best-fit Gaussian to the phase-average pulsar data at intensity levels of $10,20,40,80,160,320$, and $640 \mathrm{cts} /$ pixel illustrating the level of impact of the PSF at the site of the knot-i.e., slight but non-negligible. The intensity level is shown by the grayscale bar on the right.

November, with an amplitude and timescale comparable to those of the 2013 October incursion that we observed. Like the 2013 October incursion, the 2005 November incursion coincided with an increase in the inner knot's optical flux. While the significance of the polarization change was marginal based upon the authors' conservative statistical analysis, we conclude that optical polarization measurements may be useful for seeking a relationship between $\gamma$-ray flaring and inner knot behavior.
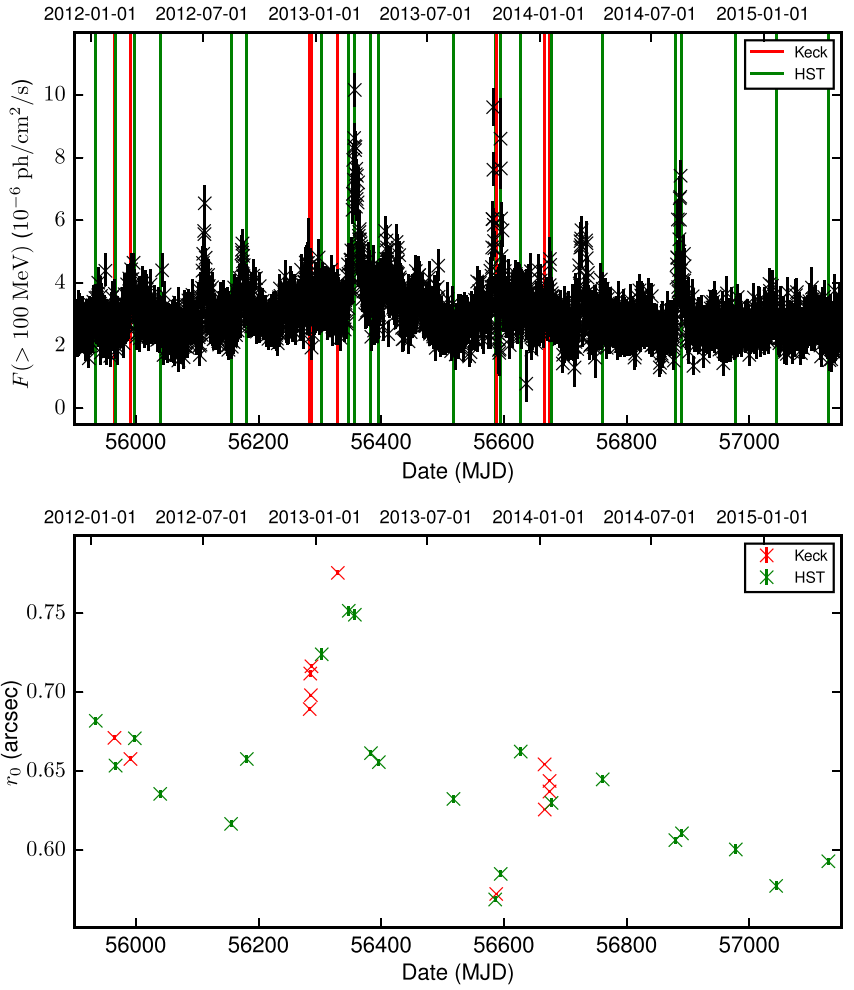

Figure 7. Fermi/LAT $12 \mathrm{hr}$ average flux (upper panel) and pulsar-knot separation (lower panel) vs. time, for Keck (red) and HST (green) observations.

\section{IMPLICATIONS FOR THEORETICAL MODELS}

In the basic model of the Crab Nebula (e.g., Rees \& Gunn 1974), the pulsar wind passes through a shock at a radius $\sim 3 \times 10^{17} \mathrm{~cm}$ where the wind momentum flux balances the nebular pressure. However, the wind should be anisotropic. For example, the momentum flux in a split-monopole $\propto \sin ^{2} \theta$ (Bogovalov 1999) and recent simulations suggest $\propto \sin ^{4} \theta$ (Tchekhovskoy et al. 2013, 2015) where $\theta$ is the polar angle. 
Table 9

Fermi/LAT 12 hr-average Fluxes and Pulsar-Knot Separations as Measured with Keck and HST (SVD Analysis)

\begin{tabular}{|c|c|c|c|c|}
\hline \# & Date & $\begin{array}{l}t_{\text {mid }} \\
\text { MJD }\end{array}$ & $\begin{array}{l}r_{0} \\
\prime \prime\end{array}$ & $\begin{array}{c}\text { Fermi } / \text { LAT } \geqslant 100 \mathrm{MeV} \\
10^{-6} \mathrm{ph} \mathrm{s}^{-1} \mathrm{~cm}^{-2}\end{array}$ \\
\hline H1 & 2012 Jan 08 & 55934.797 & $0.6819 \pm 0.0023$ & $2.74(+0.42,-0.39)$ \\
\hline K1 & 2012 Feb 08 & 55965.280 & $0.6711 \pm 0.0015$ & $2.64(+0.44,-0.40)$ \\
\hline $\mathrm{H} 2$ & 2012 Feb 10 & 55967.171 & $0.6535 \pm 0.0024$ & $2.02(+0.43,-0.39)$ \\
\hline K2 & 2012 Mar 05 & 55991.311 & $0.6577 \pm 0.0015$ & $2.70(+0.38,-0.36)$ \\
\hline $\mathrm{H} 3$ & 2012 Mar 12 & 55998.414 & $0.6708 \pm 0.0022$ & $3.70(+0.43,-0.40)$ \\
\hline $\mathrm{H} 4$ & 2012 Apr 22 & 56039.850 & $0.6356 \pm 0.0024$ & $2.40(+0.50,-0.46)$ \\
\hline H5 & 2012 Aug 16 & 56155.336 & $0.6166 \pm 0.0020$ & $2.87(+0.44,-0.44)$ \\
\hline H6 & 2012 Sep 10 & 56180.960 & $0.6577 \pm 0.0024$ & $3.06(+0.37,-0.37)$ \\
\hline K3 & 2012 Dec 22 & 56283.381 & $0.6893 \pm 0.0011$ & $2.64(+0.45,-0.45)$ \\
\hline K4 & 2012 Dec 23 & 56284.403 & $0.7118 \pm 0.0021$ & $4.56(+0.53,-0.50)$ \\
\hline K5 & 2012 Dec 24 & 56285.379 & $0.6981 \pm 0.0007$ & $3.59(+0.50,-0.45)$ \\
\hline K6 & 2012 Dec 25 & 56286.370 & $0.7164 \pm 0.0010$ & $1.89(+0.43,-0.39)$ \\
\hline $\mathrm{H} 7$ & 2013 Jan 10 & 56302.992 & $0.7240 \pm 0.0035$ & $2.68(+0.51,-0.51)$ \\
\hline K7 & 2013 Feb 06 & 56329.248 & $0.7756 \pm 0.0013$ & $3.17(+0.50,-0.50)$ \\
\hline $\mathrm{H} 8$ & 2013 Feb 24 & 56347.055 & $0.7516 \pm 0.0031$ & $4.29(+0.55,-0.51)$ \\
\hline H9 & 2013 Mar 06 & 56357.023 & $0.7491 \pm 0.0033$ & $10.88(+0.47,-0.47)$ \\
\hline $\mathrm{H} 10$ & 2013 Apr 01 & 56383.320 & $0.6614 \pm 0.0019$ & $4.05(+0.50,-0.50)$ \\
\hline H11 & 2013 Apr 14 & 56396.113 & $0.6557 \pm 0.0021$ & $3.43(+0.50,-0.50)$ \\
\hline H12 & 2013 Aug 13 & 56517.710 & $0.6324 \pm 0.0023$ & $2.56(+0.38,-0.35)$ \\
\hline H13 & 2013 Oct 20 & 56585.793 & $0.5687 \pm 0.0019$ & $3.83(+0.23,-0.23)$ \\
\hline K8 & 2013 Oct 22 & 56587.443 & $0.5722 \pm 0.0004$ & $0.70(+1.41,-0.51)$ \\
\hline H14 & 2013 Oct 29 & 56594.700 & $0.5849 \pm 0.0022$ & $7.08(+0.79,-0.79)$ \\
\hline H15 & 2013 Dec 01 & 56627.273 & $0.6624 \pm 0.0026$ & $2.76(+0.47,-0.45)$ \\
\hline K9 & 2014 Jan 09 & 56666.316 & $0.6543 \pm 0.0006$ & $3.14(+0.40,-0.40)$ \\
\hline K10 & 2014 Jan 09 & 56666.438 & $0.6257 \pm 0.0003$ & $3.13(+0.39,-0.37)$ \\
\hline K11 & 2014 Jan 17 & 56674.356 & $0.6371 \pm 0.0008$ & $4.29(+0.46,-0.45)$ \\
\hline $\mathrm{K} 12$ & 2014 Jan 17 & 56674.400 & $0.6438 \pm 0.0009$ & $3.93(+0.39,-0.37)$ \\
\hline H16 & 2014 Jan 20 & 56677.530 & $0.6299 \pm 0.0026$ & $3.37(+0.37,-0.37)$ \\
\hline H17 & 2014 Apr 13 & 56760.900 & $0.6448 \pm 0.0024$ & $3.84(+0.52,-0.52)$ \\
\hline H18 & 2014 Aug 10 & 56879.375 & $0.6063 \pm 0.0022$ & $2.50(+0.41,-0.41)$ \\
\hline H19 & 2014 Aug 21 & 56890.094 & $0.6106 \pm 0.0023$ & $3.56(+0.26,-0.25)$ \\
\hline $\mathrm{H} 20$ & 2014 Nov 16 & 56977.820 & $0.6004 \pm 0.0029$ & $2.50(+0.43,-0.39)$ \\
\hline $\mathrm{H} 21$ & 2015 Jan 21 & 57043.620 & $0.5773 \pm 0.0028$ & $3.05(+0.36,-0.34)$ \\
\hline $\mathrm{H} 22$ & 2015 Apr 16 & 57128.670 & $0.5929 \pm 0.0022$ & $2.62(+0.43,-0.43)$ \\
\hline
\end{tabular}

As a result, the shock is likely to be quite oblate. The sections of the shock near the poles would be oblique and much closer to the pulsar than the equatorial part. The observed radiation presumably comes from relativistic particles (electrons or positrons) accelerated behind the shock. Since the outflow from an oblique shock can remain relativistic, we would be able to see a compact, emitting feature (the inner knot) if the relativistic outflow happens to be aligned with our line of sight so that its emission is beamed. The inner knot should appear to us as having some offset from the pulsar due to the deflection of the outflow from its presumed radial motion (Figure 10). If the shock is approximately axisymmetric as one would expect, the projected emitting site would fall on the projected symmetry axis, leading to an alignment with the jet. The shape of the inner knot should also be more or less symmetric about the axis; whether it is elongated parallel or perpendicular to the axis depends on the geometry of the oblique shock. Furthermore, the synchrotron emission should be linearly polarized (Komissarov \& Lyutikov 2011; Y. Yuan \& R. Blandford 2015, in preparation).

The shock model predicts that the scaling between the observed properties of the knot-projected pulsar-knot separation $r_{0}$, tangential angular width $\mathrm{FWHM}_{t}$, radial angular width $\mathrm{FWHM}_{r}$, and surface brightness $S_{\mathrm{k}}$ - should be determined by the upstream magnetization $\sigma \equiv B_{1}^{2} / \mu_{0} n_{1} \gamma_{1}^{2} m c^{2}$ (where $m$ is electron mass and $B_{1}, n_{1}, \gamma_{1}$ are upstream magnetic field, proper density, bulk Lorentz factor, respectively) plus three more parameters that characterize the shape of the shock near the emitting site: the unprojected pulsar-knot separation $r_{\mathrm{k}}$; the incident angle $\delta_{1}$, defined to be the angle between upstream velocity and the shock surface as shown in Figure 10; and the shock poloidal radius of curvature $R_{\mathrm{c}}$.

Let the outflow be deflected from the radial direction by an angle $\Delta$ at the shock. We then have $r_{0}=r_{\mathrm{k}} \Delta$. The knot tangential size is determined by the Doppler beaming and the shock radius of curvature in the toroidal direction as $\mathrm{FWHM}_{t} \approx 2 r_{\mathrm{k}} / \gamma$, where $\gamma$ is the downstream Lorentz factor. In the simplest shock model with an isotropic plasma, both $\Delta$ and $\gamma$ are functions of $\sigma$ and $\delta_{1}: \Delta=\delta_{1}-\arctan \left(\chi \tan \delta_{1}\right)$ and $\gamma=1 /\left(\sin \delta_{1} \sqrt{1-\chi^{2}}\right)$, where $\chi \equiv v_{2} \perp v_{1 \perp}=B_{1} / B_{2}=$ $\left(1+2 \sigma+\sqrt{16 \sigma^{2}+16 \sigma+1}\right) / 6(1+\sigma)$ is the compression ratio at the shock, $v_{1,2 \perp}$ being the component of upstream/ downstream velocity perpendicular to the shock (Komissarov \& Lyutikov 2011). The radial knot size $\mathrm{FWHM}_{r}$ should also be proportional to $r_{\mathrm{k}} / \gamma$ but will have an additional factor involving the ratio $R_{\mathrm{c}} / r_{\mathrm{k}}$.

As to the intensity, we assume that the emitting particles have a power-law distribution $\propto n^{\prime} \gamma^{\prime-p}$ in the fluid rest frame, where $n^{\prime}$ and $\gamma^{\prime}$ are particle density and Lorentz factor in the 

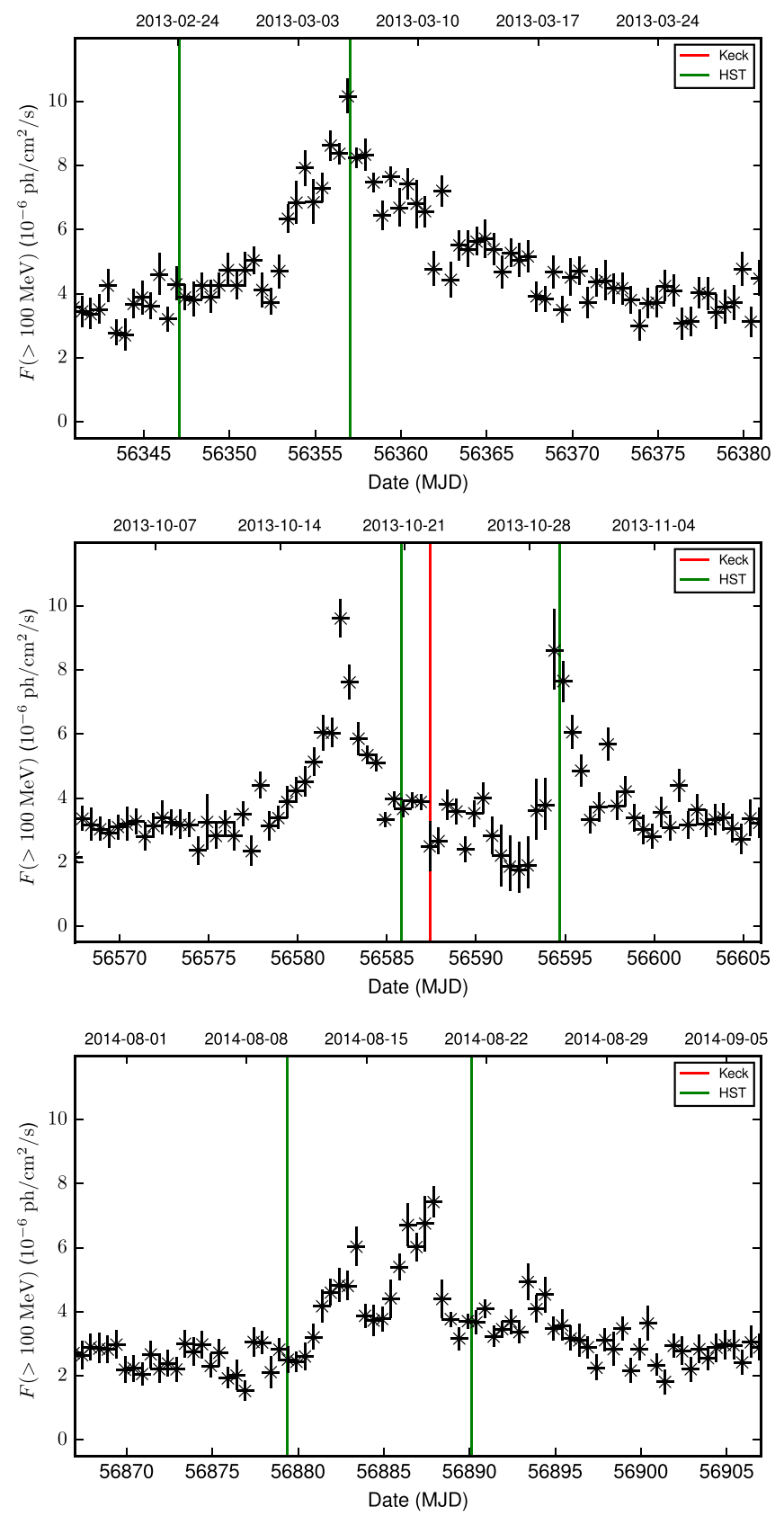

Figure 8. Fermi/LAT 12 hr average fluxes during Keck (red) and HST (green) observations at the time of the largest $\gamma$-ray flares in 2013 March (top), in 2013 October (middle), and in 2014 August (bottom).

same reference frame, and $p \sim 2.6$ from the measured spectral index of the knot (Sollerman 2003; Melatos et al. 2005).

The emissivity in the fluid rest frame is $j_{\nu^{\prime}}^{\prime}\left(\nu^{\prime}\right) \propto$ $n^{\prime} B^{\prime(p+1) / 2} \nu^{\prime-(p-1) / 2}$, where $B^{\prime}$ and $\nu^{\prime}$ are the magnetic field and the radiation frequency measured in this reference frame. After transforming to the nebula frame, the emissivity has a dependence on the flow Lorentz factor $\gamma$, particle (improper) number density $n$, and magnetic field $B$ as follows: $j_{\nu} \propto \mathcal{D}^{2+(p-1) / 2}(n / \gamma)(B / \gamma)^{(p+1) / 2}$, where $\mathcal{D}=\nu / \nu^{\prime}=$ $\sqrt{1-\beta^{2}} /(1-\boldsymbol{\beta} \cdot \boldsymbol{n})$ is the Doppler factor, in which $\boldsymbol{\beta}$ is the flow velocity and $\boldsymbol{n}$ is a unit vector along the direction of the line of sight. The surface brightness corresponds to the emissivity integrated along the line of sight in the nebular frame: $S_{\nu}=\int j_{\nu} d l$. To estimate the peak surface brightness,

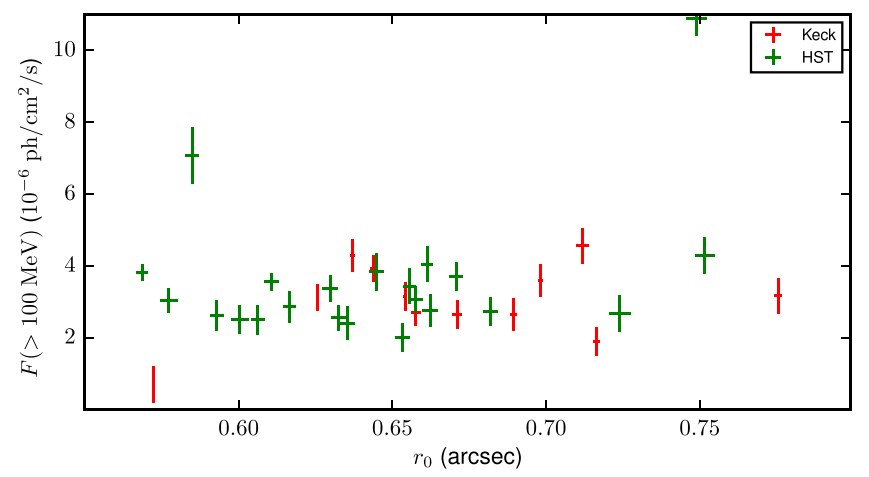

Figure 9. Fermi/LAT 12-hr-average fluxes centered on the times of Keck (red) and $H S T$ (green) measurements of pulsar-knot separations.

one takes the maximum Doppler factor $\mathcal{D} \approx 2 \gamma$, and we get $S_{\nu \text {, peak }} \propto n B^{(p+1) / 2} \ell$ where $\ell$ is an estimation of the length of the emitting region along the line of sight. As roughly $B \propto r_{\mathrm{k}}^{-1}$ and $n \propto r_{\mathrm{k}}^{-2}$, we have $S_{\nu \text {,peak }} \propto r_{\mathrm{k}}^{-2-(p+1) / 2} \ell$.

These previous relations allow us to set the constraints discussed below using both the steady and variable properties of the knot.

\subsection{Variability}

In the shock model, variability can arise either upstream or downstream of the shock. First, stress tensor variation in the nebula can cause the shock radius and shape to vary over time. Three-dimensional MHD simulations (Porth et al. 2014) show that even if the upstream condition is fixed, the post-shock flow can be quite variable: there is vortex-shedding from the termination shock, and the shock constantly interacts with waves and vortices in the nebula. Those variations are expected to be mostly magnetic in origin, and the interactions between the shock and the downstream flow are quite nonlinear. The picture is similar to earlier 2D simulations (e.g., Camus et al. 2009) except that the kink instability sets in so that the hoop compression is less prominent as in the 2D case; also shortterm variability is less pronounced. The $3 \mathrm{D}$ simulations give a typical variation timescale of less than one year.

Now suppose that the change of downstream pressure causes the shock radius $r_{\mathrm{k}}$ to change. For simplicity we first assume that $\Delta, \ell, \gamma$, and $p$ stay more or less the same, then from the above scaling relations, we have $S_{\nu \text {,peak }} \propto r_{0}^{-2-(p+1) / 2}, \mathrm{FWHM}_{t} \propto r_{0}$, and knot flux $F_{\nu} \propto S_{\nu, \text { peak }} \mathrm{FWHM}_{t} \mathrm{FWHM}_{r} \propto r_{0}^{-(p+1) / 2}$, assuming $\mathrm{FWHM}_{r}$ to also be roughly proportional to $r_{\mathrm{k}}$. In reality $\Delta, \ell$, and $\gamma$ change with the shock radius $r_{\mathrm{k}}$ as well, as the shock shape and upstream incident angle $\delta_{1}$ vary. Consequently, the observed correlation is more complicated and less clean; however, the general trend is consistent with our expectation of the shock model. In the 3D simulation of Porth et al. (2014), the synthetic images of the knot show a variation consistent with the observations of Moran et al. (2013), suggesting that downstream variation of the shock could provide a good explanation for the knot variability.

Variations initiated by the pulsar can also cause the knot properties to change. In such a scenario, the shortest possible variation timescale is $t_{v} \sim r_{\mathrm{k}}(1-\cos (1 / \gamma)) / c \sim r_{\mathrm{k}} /\left(2 \gamma^{2} c\right)$ $\sim 1$ day $\left(r_{\mathrm{k}} / 10^{17} \mathrm{~cm}\right)(\gamma / 5)^{-2}$ up to $10 \%$ inaccuracy assuming $\gamma \gtrsim 3$. However, there is no evidence in the pulsar timing that this is actually happening. 


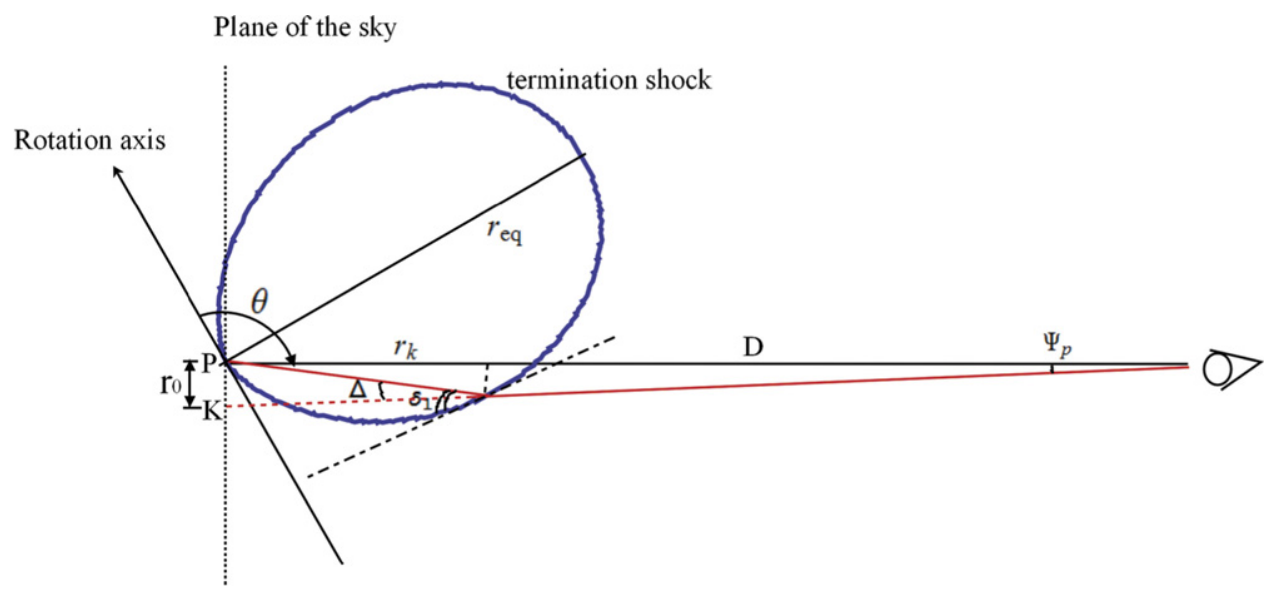

Figure 10. Geometry of the termination shock on the meridional plane determined by the pulsar rotation axis and the observer's line of sight toward the pulsar (point $\mathrm{P})$. The angle between the line of sight and the pulsar rotation axis is $\theta_{\mathrm{ob}}=2 \pi / 3$, consistent with that measured by Ng \& Romani (2008). The inner knot (point $\mathrm{K}$ ) is projected on the plane of the sky at an angle of $\Psi_{\mathrm{p}}=r_{0} / D \approx 0$." 65 southeast of the pulsar. (NB: Throughout this paper, $r_{0}$ appears in units of arcseconds.) The angle between the upstream velocity and the shock surface is $\delta_{1}$; the outflow is deflected from the radial direction by an angle $\Delta$.

\subsection{Time-averaged Properties of the Knot}

The properties of the knot may be used to set constraints on the flow composition and how much dissipation occurs at the shock. One constraint comes from geometrical relations among the three measured angles $r_{0}, \mathrm{FWHM}_{t}$, and $\mathrm{FWHM}_{r}$. For the simplest shock model, we have $r_{0}=r_{\mathrm{k}} \Delta$ and FWHM $_{t} \approx r_{\mathrm{k}} / \gamma$, where $\Delta$ and $\gamma$ only depend on $\sigma$ and $\delta_{1}$, such that the ratio $\mathrm{FWHM}_{t} / r_{0}$ sets limit on $\sigma$ and $\delta_{1}$. We find that $\mathrm{FWHM}_{t} / r_{0}=2 /(\gamma \Delta)=2 \sin \delta_{1} \sqrt{1-\chi^{2}} /$ $\left[\delta_{1}-\arctan \left(\chi \tan \delta_{1}\right)\right] \geqslant 2.8$, with the minimum at $\sigma=0$ and $\delta_{1}=0.39$. In contrast, the observation gives $\mathrm{FWHM}_{t} \sim 0.5 r_{0}$, indicating that some important ingredient is missing in the standard shock model. For example, the upstream flow might have already been bent before arriving at the shock; and/or the anisotropic particle distribution downstream might be important in determining the size of the knot. Although more detailed investigations will be described elsewhere, the above discussion on variability and the following discussion on energetics should be valid for quite general shock models.

The shape of the knot also has some interesting implications. In both the Hubble and Keck images, the knot is not just a simple ellipse that only involves quadratic terms of coordinates; it has curvature and appears to be convex away from the pulsar ("smile"), so at least a third moment of the coordinates is needed to describe the shape. A simple intuitive picture is based on the geometry that the shock surface is similar to a torus centered on the pulsar and we look through the hole from the bottom. If emission from the shock outflow roughly follows the local toroidal magnetic field, one would imagine the knot to be convex toward the pulsar ("frown")—contradictory to what we see. In fact, we should not overlook the possibility that the outflow may have significant bending downstream. If the outflow bends toward the equator by an angle $\sim 1 / \gamma$ within the emission length, we would see the knot more extended (contracted) on the far (near) side with respect to the pulsar, as additional flow lines enter (originally aligned flow lines leave) the emission cone on the corresponding side.

Another constraint comes from the observed flux of the knot. In the $K^{\prime}$ band, the typical knot-pulsar flux ratio is $\sim 0.063$ (Table 4). If we take the photometry of the pulsar from Sandberg \& Sollerman (2009), the pulsar $K_{s}$ band magnitude is $13.80 \pm$
0.01 , after dereddening with the best-fit value $E(B-V)=0.52$ (Sollerman et al. 2000) and the extinction law from Fitzpatrick (1999), this corresponds to a flux of $2.2 \times 10^{-26} \mathrm{erg} \mathrm{s}^{-1}$ $\mathrm{cm}^{-2} \mathrm{~Hz}^{-1}$. So the inner knot flux in $K^{\prime}$ band should be roughly $F_{\nu}^{\mathrm{knot}}=1.4 \times 10^{-27} \mathrm{erg} \mathrm{s}^{-1} \mathrm{~cm}^{-2} \mathrm{~Hz}^{-1}$. Similarly, optical data (Table 6) on average give a dereddened flux of $F_{\nu}^{\mathrm{knot}}=1 \times 10^{-27} \mathrm{erg} \mathrm{s}^{-1} \mathrm{~cm}^{-2} \mathrm{~Hz}^{-1}$. However, we do not attempt here to get a spectral index using the time-averaged fluxes due to the variability of the knot and the fact that most of the multi-wavelength observations are not simultaneous. Previous measurements by Sollerman (2003) and Melatos et al. (2005) indicate that the spectrum of the knot in IR to optical range is a power law $F_{\nu} \propto \nu^{-\alpha}$ with $\alpha \sim 0.8$, and Melatos et al. (2005) further measured that this power law extends to NUV $(160-320 \mathrm{~nm})$, with a flux in NUV $F_{\nu}^{\mathrm{knot}}=0.12 \mathrm{mJy}=$ $1.2 \times 10^{-27} \mathrm{erg} \mathrm{s}^{-1} \mathrm{~cm}^{-2} \mathrm{~Hz}^{-1}$ - this is the highest energy at which the knot has been measured. A few more recent measurements show slightly different spectral index ranging from 0.63 (Tziamtzis et al. 2009) to 1.3 (Sandberg \& Sollerman 2009), but unfortunately these are based on nonsimultaneous data. It would be very interesting to refine the spectral measurement using near simultaneous IR/optical images in the future. In what follows we adopt a fiducial spectral model $F_{\nu}^{\text {knot }} \sim 10^{-27}\left(\nu / 10^{15} \mathrm{~Hz}\right)^{-0.8} \mathrm{erg} \mathrm{s}^{-1} \mathrm{~cm}^{-2} \mathrm{~Hz}^{-1}$ with the caveat that this could change with better observations.

Using the above spectral model, we find that the knot integrated flux in the IR/optical band $\left((0.1-2) \times 10^{15} \mathrm{~Hz}\right)$ is $F_{\mathrm{O} / \mathrm{IR}}^{\mathrm{knot}}=2.6 \times 10^{-12} \mathrm{erg} \mathrm{s}^{-1} \mathrm{~cm}^{-2}$. Thus, the luminosity of the knot per steradian is $L_{\mathrm{O} / \mathrm{IR}, \Omega}=D^{2} F_{\mathrm{O} / \mathrm{IR}}^{\mathrm{knot}}=10^{32} \mathrm{erg} \mathrm{s}^{-1}$, where $D \sim 2 \mathrm{kpc}$ is the distance of the Crab. If we define $\epsilon \equiv L_{\mathrm{O} / \mathrm{IR}, \Omega} /(\dot{E} / 4 \pi)$, where $\dot{E}=5 \times 10^{38} \mathrm{erg} \mathrm{s}^{-1}$ is the pulsar spin-down power, $\epsilon$ can be used as a measure of the radiative efficiency of the shock along the direction of line of sight in IR to optical range. Here we find $\epsilon=2.5 \times 10^{-6}$ and the shock is highly adiabatic.

We can obtain an estimation of the enthalpy fraction of IR/ optical-emitting particles in the downstream flow

$$
\eta\left(\gamma^{\prime}\right)=\frac{8}{3} \frac{\gamma t_{\text {cool }}^{\prime}}{t_{\text {flow }}} \frac{\nu L_{\nu, \Omega}}{\dot{E} /(4 \pi)}=\frac{8}{3} \frac{\gamma t_{\text {cool }}^{\prime}}{t_{\text {flow }}} \frac{\nu L_{\nu, \Omega}}{\dot{E} /(4 \pi)},
$$


where $t_{\text {flow }}$ is the flow timescale and $t_{\text {cool }}^{\prime}=$ $6 \pi \epsilon_{0} m^{3} c^{3} /\left(e^{4} B^{\prime 2} \gamma^{\prime}\right) \approx 10^{2} \gamma^{2} B_{-3}^{-3 / 2} \nu_{14}^{-1 / 2}$ years is the synchrotron cooling time of the particles in the fluid rest frame (we have adopted $B=B_{-3} \mathrm{mG}$ and $\nu=\nu_{14} 10^{14} \mathrm{~Hz}$ for the numerical value). Thus the particle injection rate per unit steradian in the corresponding energy band is

$$
\dot{N}\left(\gamma^{\prime}\right)=\frac{3 \eta\left(\gamma^{\prime}\right)}{4 \gamma^{\prime} m c^{2}} \frac{\dot{E}}{4 \pi \gamma}
$$

Adopting typical values $\gamma=5 \gamma_{5}, t_{\text {flow }}=r_{17} 10^{17} \mathrm{~cm} / c$, we find that for IR-emitting particles, $\eta_{\mathrm{IR}}=0.05 \gamma_{5}^{3} B_{-3}^{-3 / 2} r_{17}^{-1}$, and $\dot{N}_{\text {IR }}=2 \times 10^{36} \gamma_{5}^{2} B_{-3}^{-1} r_{17}^{-1} \mathrm{~s}^{-1} \mathrm{sr}^{-1}$. The spectral index of the knot indicates that the particle distribution power-law index should be $p \sim 2.6$, thus the IR-emitting particles should comprise the majority of particle pressure downstream. From the above estimations, it seems that the particle injection rate at the shock is marginally consistent with the scenario that most of the IR/optical-emitting particles are provided by the shock.

Regarding particle acceleration mechanisms, we notice that IR-emitting particles usually go through $N=t_{\text {flow }} \nu_{g}$ Larmor orbits, where $\nu_{g}=\nu_{B} / \gamma^{\prime}$ is the relativistic gyrofrequency-the cyclotron frequency $\left(\nu_{B}\right)$ divided by the electron's Lorentz factor $\left(\gamma^{\prime}\right)$. Eliminating $\gamma^{\prime}$ in favor of the magnetic field $(B)$ and observing frequency $(\nu)$, the number of orbits within the flow timescale is $N \approx 6 \times 10^{4} B_{-3}^{3 / 2} r_{17} \nu_{14}^{-1 / 2}$. To allow sufficient time for acceleration mechanisms requiring instabilities or stochastic processes to operate, requires that $N>>1$. However, for particles emitting $\gamma$ rays of energy $300 \mathrm{MeV}$, $N \lesssim 1$, thus requiring special acceleration mechanisms-if the emitting electrons are indeed accelerated at the shock.

One more constraint comes from the polarization of the knot. Most recent polarimetry performed by Moran et al. (2013) gives a high polarization degree of $\sim 60 \%$, with position angle aligned with the symmetry axis, indicating a strongly toroidal magnetic field. Here we need to be careful about the depolarization effect due to relativistic kinematics (Lyutikov et al. 2003). What happens then is that Lorentz transformation of the emission of a relativistic plasma from the comoving frame to the lab frame produces a rotation of the polarization vector. For a curved emitting surface, neighboring fluid elements have slightly different velocities, thus their polarization vectors experience different amounts of rotation. As one sums the contribution from the visible surface (this is essentially what we observe), the result is some degree of depolarization. We find that for the oblique shock in the Crab Nebula, under an ultrarelativistic approximation, the upper limit of polarization degree is similar to that in Lyutikov et al. (2003): $56.25 \%$ for particle spectral index $p=3$ and $43.4 \%$ for $p=2$. Thus, the observed high degree of polarization and its possible variation are highly constraining on theoretical models. Mildly relativistic outflow may give a higher polarization degree but may not produce a small enough knot (see Porth et al. 2014). In addition, particle acceleration mechanisms that generate turbulence could further depolarize the emission. It would be interesting to repeat this polarization observation, especially with $\mathrm{AO}$.

\section{CONCLUSIONS}

We have (1) introduced a new approach for analyzing time series images; (2) discovered that the key properties of the knot (radial width, tangential width, flux) are correlated with the time-variable separation between the knot and the pulsar; (3) shown that with the available data it is not possible to determine a strong correlation between the knot separation and the occurrence of $\gamma$-ray flares; (4) set an upper limit to the lowenergy X-ray flux from the inner knot; and (5) discussed the implications of our observations to set constraints on particular elements of the standard shock modeling of the relativistic outflow from the pulsar. These include inferences as to wind magnetization $\sigma$, shock shape parameters such as incident angle $\delta_{1}$, and poloidal radius of curvature $R_{\mathrm{c}}$, as well as the IR/ optical-emitting particle enthalpy fraction. We found that while the standard shock model gives good agreements with observations in many aspects, there remain two puzzles: (a) the angular size of the knot relative to the pulsar-knot separation is much smaller than expected; and (b) the variable yet high degree of polarization is difficult to reconcile with a highly relativistic outflow (see, however, Porth et al. 2014). We also found that the IR/optical flux of the inner knot is marginally consistent with the scenario in which the shock accelerates most of the optical-emitting particles in the nebula.

The Fermi/LAT Collaboration acknowledges generous ongoing support from a number of agencies and institutes that have supported both the development and the operation of the LAT as well as scientific data analysis. These include the National Aeronautics and Space Administration and the Department of Energy in the United States, the Commissariat à l'Energie Atomique and the Centre National de la Recherche Scientifique/Institut National de Physique Nucléaire et de Physique des Particules in France, the Agenzia Spaziale Italiana and the Istituto Nazionale di Fisica Nucleare in Italy, the Ministry of Education, Culture, Sports, Science, and Technology (MEXT), High Energy Accelerator Research Organization (KEK), and the Japan Aerospace Exploration Agency (JAXA) in Japan, and the K. A. Wallenberg Foundation, the Swedish Research Council, and the Swedish National Space Board in Sweden. Additional support for science analysis during the operations phase is gratefully acknowledged from the Istituto Nazionale di Astrofisica and the Centre d'Etudes Spatiales in France. The research leading to these results has also received funding from the European Commission Seventh Framework Programme (FP7/20072013) under grant agreement n. 267251. Several of the authors would also like to acknowledge both funding and solid support from the Chandra X-ray Center and the Hubble Space Telescope Science Institute under a number of observing proposals: GO3-14054Z, GO3-14057Z, GO4-15058Z, GO415059Z, GO-13109, GO-13196, and GO-13348.

\section{APPENDIX \\ DATA ANALYSIS}

\section{A.1. Keck}

To study the knot using the high-resolution Keck adaptive optics (AO) data, we characterize the region around the pulsar using an empirically measured PSF and an analytical model of the knot geometry. 
Pulsar \& Knot

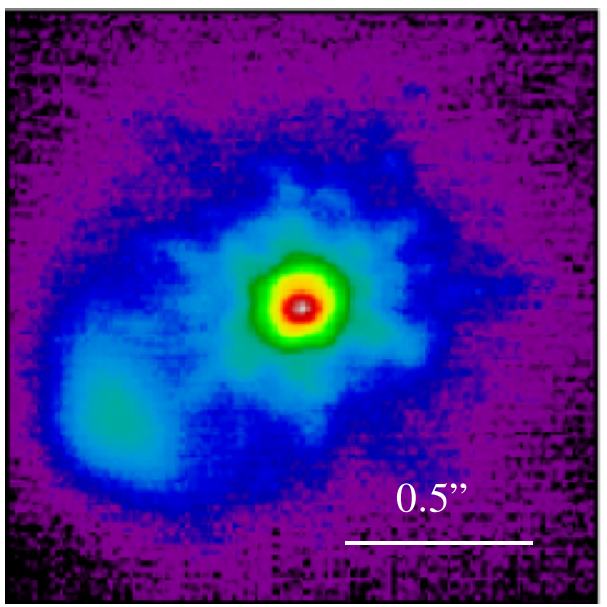

PRF Star

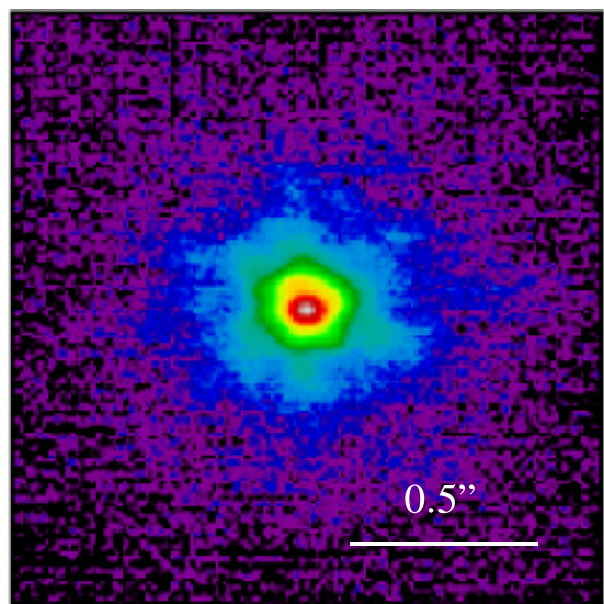

K8: 2013-10-22 NIRC2-narrow

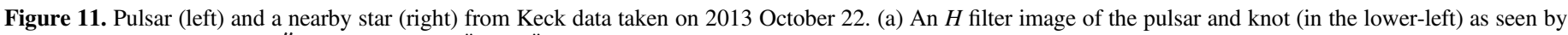

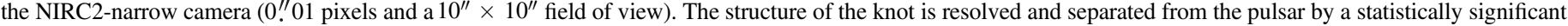
valley. (b) A nearby $\left(5^{\prime \prime}\right)$ comparison star, used to establish the point-response-function. Both images are shown with a logarithmic color stretch.

Table 10

Results of the Traditional Analysis of the First 17 HST Observations

\begin{tabular}{|c|c|c|c|c|}
\hline$\#$ & $\begin{array}{c}r_{0} \\
\prime \prime\end{array}$ & $\underset{\prime \prime}{\mathrm{FWRMS}_{r}}$ & $\begin{array}{c}\mathrm{FWRMS}_{t} \\
\mathrm{FW}\end{array}$ & $\begin{array}{l}F_{\mathrm{k}} \\
\mathrm{e} / \mathrm{s}\end{array}$ \\
\hline H1 & $0.660 \pm 0.012$ & $0.287 \pm 0.019$ & $0.551 \pm 0.037$ & $116.5 \pm 2.9$ \\
\hline $\mathrm{H} 2$ & $0.634 \pm 0.012$ & $0.283 \pm 0.020$ & $0.538 \pm 0.038$ & $110.9 \pm 2.8$ \\
\hline $\mathrm{H} 3$ & $0.649 \pm 0.013$ & $0.333 \pm 0.021$ & $0.594 \pm 0.038$ & $131.6 \pm 3.3$ \\
\hline $\mathrm{H} 4$ & $0.615 \pm 0.012$ & $0.255 \pm 0.020$ & $0.489 \pm 0.038$ & $90.4 \pm 2.3$ \\
\hline H5 & $0.588 \pm 0.012$ & $0.278 \pm 0.019$ & $0.560 \pm 0.038$ & $112.9 \pm 2.8$ \\
\hline H6 & $0.635 \pm 0.012$ & $0.290 \pm 0.020$ & $0.552 \pm 0.037$ & $111.6 \pm 2.8$ \\
\hline $\mathrm{H} 7$ & $0.706 \pm 0.010$ & $0.288 \pm 0.021$ & $0.589 \pm 0.044$ & $97.6 \pm 2.4$ \\
\hline $\mathrm{H} 8$ & $0.734 \pm 0.017$ & $0.336 \pm 0.024$ & $0.658 \pm 0.046$ & $109.2 \pm 2.7$ \\
\hline $\mathrm{H} 9$ & $0.732 \pm 0.013$ & $0.319 \pm 0.022$ & $0.650 \pm 0.046$ & $108.1 \pm 2.7$ \\
\hline $\mathrm{H} 10$ & $0.641 \pm 0.009$ & $0.299 \pm 0.020$ & $0.580 \pm 0.039$ & $117.6 \pm 2.9$ \\
\hline H11 & $0.637 \pm 0.011$ & $0.314 \pm 0.020$ & $0.561 \pm 0.036$ & $129.2 \pm 3.2$ \\
\hline H12 & $0.613 \pm 0.012$ & $0.258 \pm 0.017$ & $0.578 \pm 0.039$ & $121.3 \pm 3.0$ \\
\hline H13 & $0.547 \pm 0.009$ & $0.224 \pm 0.015$ & $0.491 \pm 0.033$ & $119.2 \pm 3.0$ \\
\hline H14 & $0.552 \pm 0.009$ & $0.236 \pm 0.015$ & $0.511 \pm 0.033$ & $124.8 \pm 3.1$ \\
\hline H15 & $0.631 \pm 0.012$ & $0.268 \pm 0.018$ & $0.588 \pm 0.040$ & $115.2 \pm 2.9$ \\
\hline H16 & $0.614 \pm 0.011$ & $0.266 \pm 0.025$ & $0.519 \pm 0.049$ & $116.9 \pm 2.9$ \\
\hline H17 & $0.624 \pm 0.012$ & $0.259 \pm 0.024$ & $0.549 \pm 0.050$ & $127.8 \pm 3.2$ \\
\hline
\end{tabular}

\section{A.1.1. PSF Subtraction}

We subtracted the PSF of the pulsar using a nearby PSF star (Figure 11). During our observations, the laser guide star, and thus the center of the anisoplanatic patch, was aimed at the point halfway between the PSF star and the pulsar. We fit the PSF star, a background plus a power-law based seeing-disk, to the pulsar. For the fit, we excluded the region in the pulsar subimage that surrounds the knot and used the other $270^{\circ}$. The background was fit with a sloping plane model to allow for the variable nebular background in the pulsar and PSF star subimages. The fitting was performed with a Levenburg-Marquadt fitter.

\section{A.1.2. Deconvolution}

We chose not to deconvolve the knot with the empirically measured PSF. Tests on several images showed that
Table 11

Results of Regression Analyses for the First 17 HST Observations Based on the Traditional Data Analysis

\begin{tabular}{lccrc}
\hline \hline $\begin{array}{l}\text { Power-law for } \\
r_{1}=0 \text {." } 6342\end{array}$ & & & & \\
& & & & \\
Property & Unit & $p\left(r_{1}\right)$ & Power-law & \\
\hline FWRMS $_{r}$ & $\prime \prime$ & $0.2801 \pm 0.0046$ & $1.14 \pm 0.21$ & $6.0 \mathrm{E}-5$ \\
FWRMS $_{t}$ & $\prime \prime$ & $0.5605 \pm 0.0067$ & $0.85 \pm 0.15$ & $4.9 \mathrm{E}-5$ \\
$F_{\mathrm{k}}$ & $\mathrm{e} / \mathrm{s}$ & $114.8 \pm 2.6$ & $-0.39 \pm 0.29$ & $1.9 \mathrm{E}-1$ \\
\hline
\end{tabular}

deconvolution of the shape of the knot made insignificant differences to the fit values and uncertainty.

\section{A.1.3. Knot Model}

To fit the geometric parameters of the knot, we masked out the center diffraction-limited core of the pulsar, and used a Levenburg-Marquadt fitting technique to fit a two-dimensional Gaussian to the shape of the Knot.

We used a model of the form $S(r, \psi)=S_{\mathrm{b}}+$ $S_{\mathrm{k}} \exp \left(-\frac{1}{2}\left(\left(r-r_{0}\right)^{2} / \sigma_{r}^{2}+\left(\psi-\psi_{0}\right)^{2} / \sigma_{\psi}^{2}\right)\right)$.

We also allowed the center of the coordinate system $\left(x_{0}, y_{0}\right)$ to vary slightly $(0$ " 05$)$. To determine the fit uncertainties, we used the $1 \sigma$ errors from the self-covariance of the fit parameters. Results were tabulated in Table 4.

\section{A.2. HST}

\section{A.2.1. HST-Traditional Analysis}

In order to measure the knot properties (position, flux, tangential, and radial width) we used the SExtractor package (Bertin \& Arnouts 1996). SExtractor has been extensively used for the analysis of $H S T$ data, in particular for the ACS camera (e.g., for the Hubble Ultra Deep Field project, Beckwith et al. 2006). For source detection, we require a minimum of five contiguous pixels with a detection threshold $5 \sigma$ above the rms background, with a total of 32 deblending subthresholds, and 
(a)

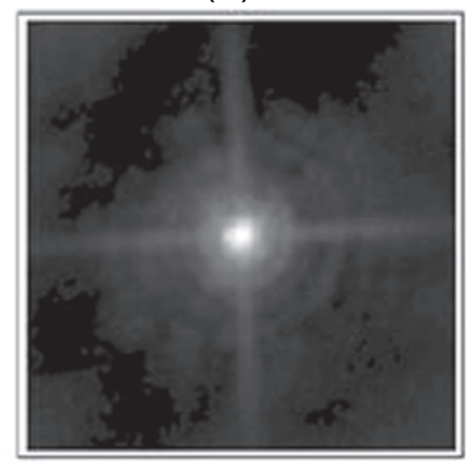

(b)

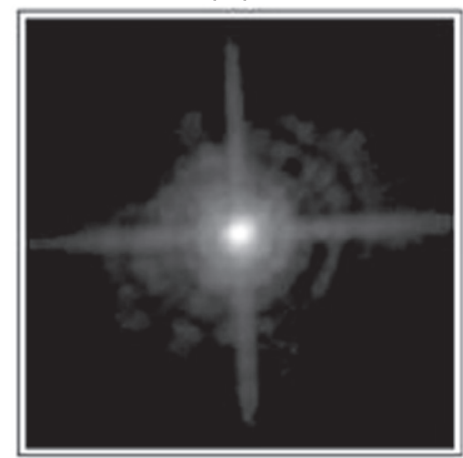

(c)

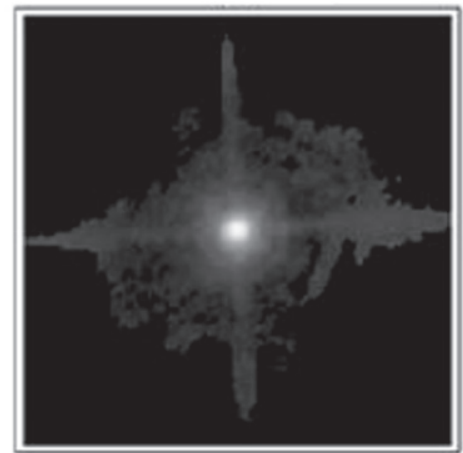

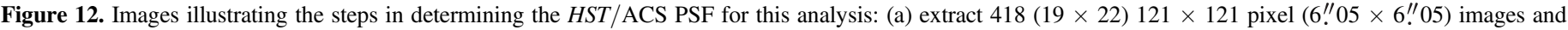

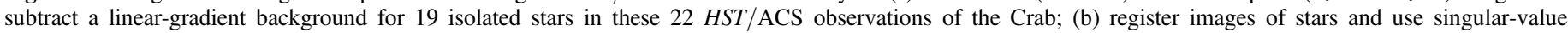

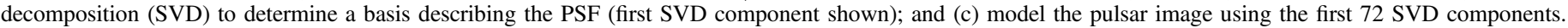

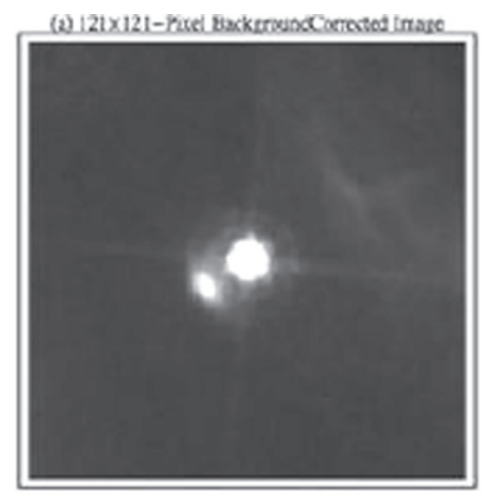

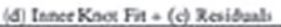

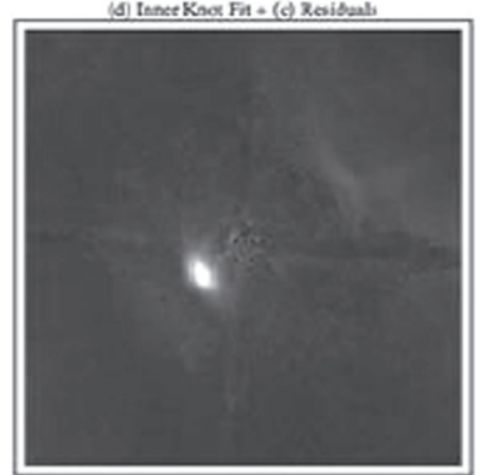

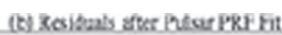

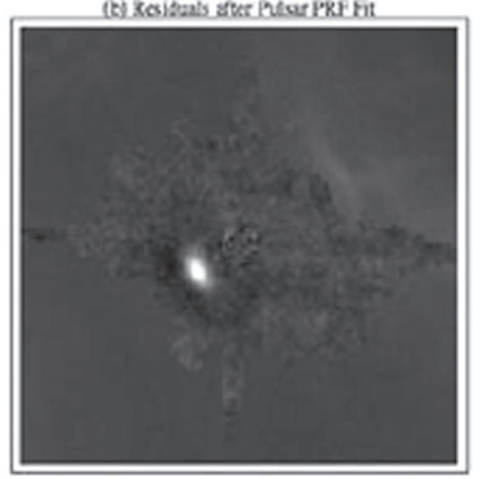

(c) Descandition of (C)

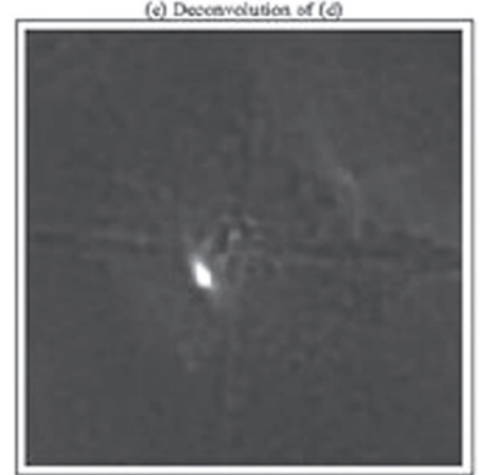

(c) Resionalb efter tmer Koos + Puka KRE Fit

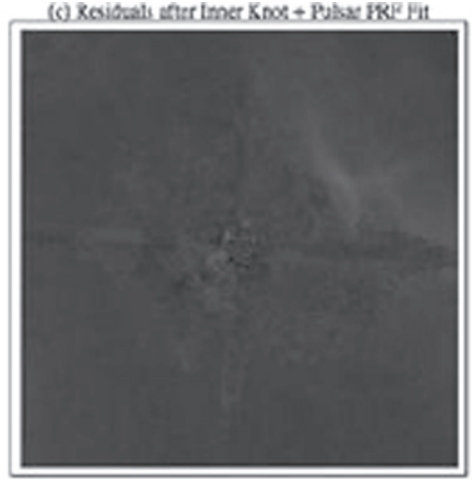

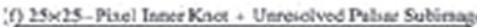

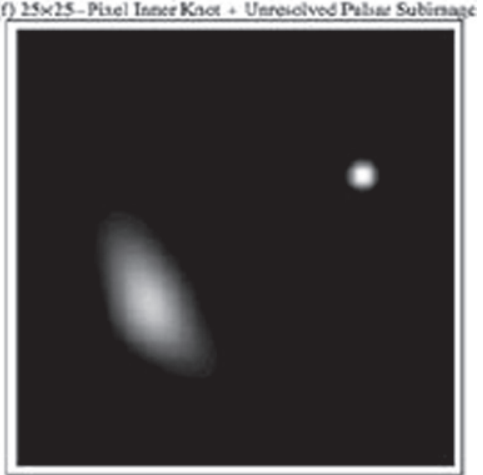

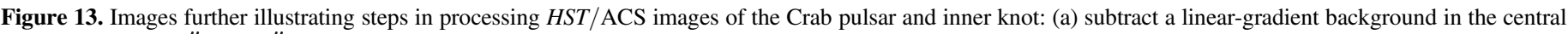

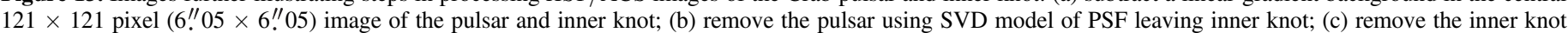

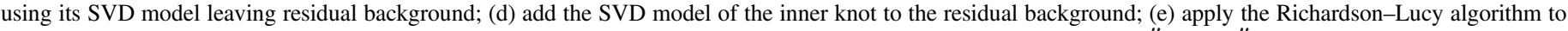

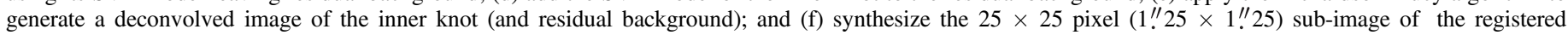
(unresolved) pulsar and SVD model of the inner knot.

with a contrast parameter of 0.005 , setting the background mesh size to $16 \times 16$ ACS pixels.

The position of a source is evaluated by SExtractor as the barycenter of the source brightness distribution. Flux is computed within an elliptical aperture, using an implementation of the method by Kron (1980). Note that the pulsar is not saturated and that diffraction spikes from the pulsar that might cross the inner knot only marginally affect its flux measurements. The parameters of this ellipse (semiaxes and orientation), evaluated using the second moments of the object's brightness distribution also yield a measure of the object morphology. Indeed, for the case of the knot, the direction of the minor axis of the ellipse turned out to be consistent with the pulsar-knot direction in all images. Thus, the minor axis and the major axis of the ellipse measure the rms full widths (FWRMS) in the radial and tangential directions, respectively.

In order to assess systematic errors, we performed simulations with the ESO/MIDAS software. ${ }^{13}$ We added to the ACS images a "synthetic knot." To generate such an artificial source, we assumed a two-dimensional Gaussian brightness distribution, with the minor axis aligned with the true pulsar-knot direction. The synthetic knot was positioned to the NW of the

\footnotetext{
${ }^{13}$ https://www.eso.org/sci/software/esomidas/
} 

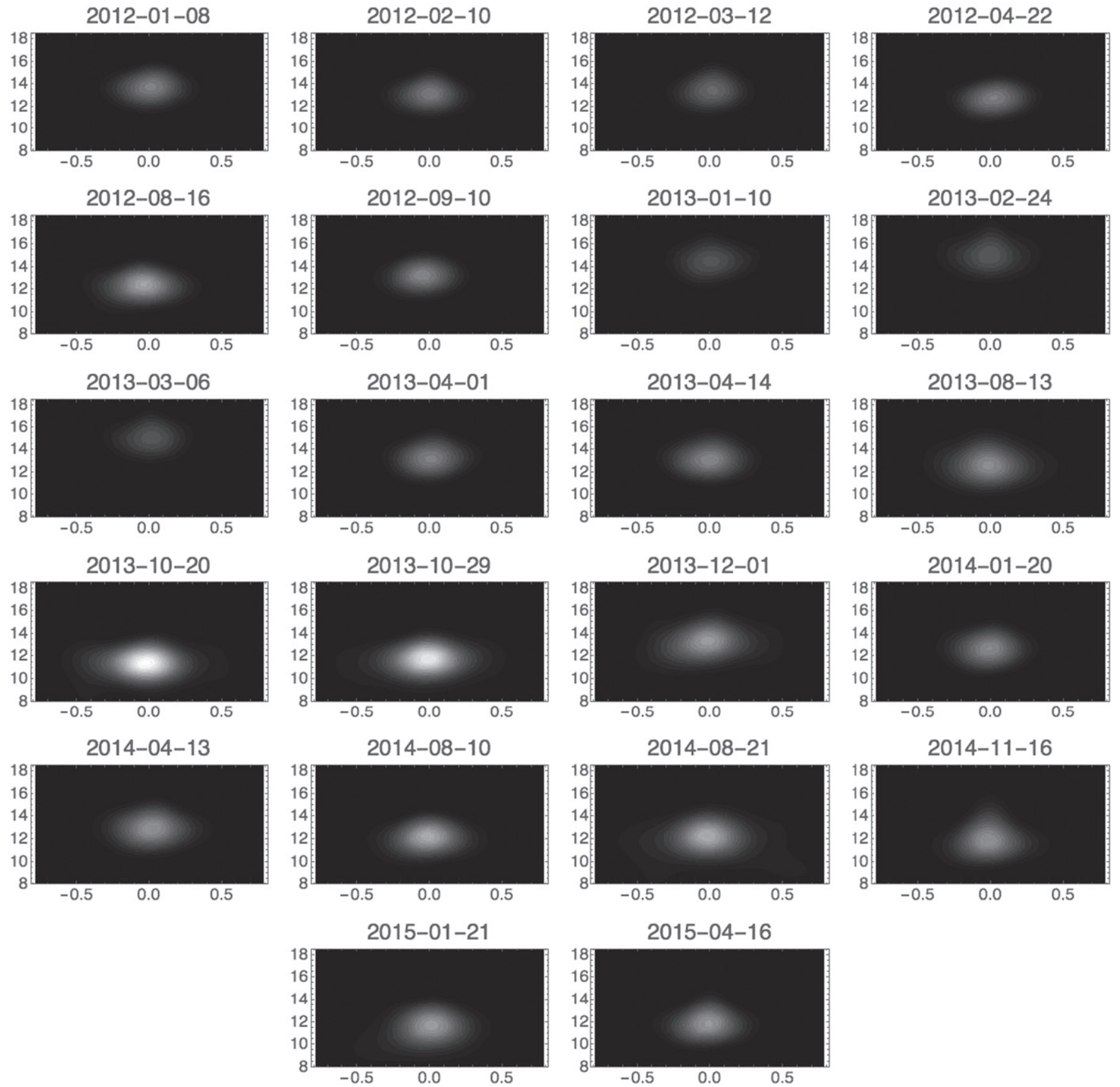


distance from the pulsar in $\left(00^{\prime \prime} 05\right)$ pixels.

pulsar, opposite but along the true pulsar-knot direction, and at an angular distance comparable to that of the true knot. We repeated the exercise by varying the flux, position, and morphology of the synthetic knot and we estimated the uncertainties in the parameters recovered using SExtractor. ${ }^{14}$

Table 10 lists the best-fit values of the model parameters of the inner knot for each of the first 17 observations based upon this traditional analysis and modeling. Table 11 shows the results of a regression analysis on the listed parameters. We obtained an additional five $H S T$ observations but did not perform the traditional analysis on those data.

\footnotetext{
${ }^{14} \mathrm{http}: / /$ www.astromatic.net/software/sextractor
}

\section{A.2.2. HST-SVD}

As the inner knot is within $1^{\prime \prime}$ of the Crab pulsar and varies in position and size, we also developed special procedures for characterizing it using the central $121 \times 121$ pixel $(6$ ". $05 \times 6$ ". 05$)$ images of the 22 HST/ACS observations. The major steps are (1) to remove the pulsar from each image, (2) to use the residual image to generate an image of the inner knot, and (3) to characterize the properties of the inner knot.

\section{A.2.2.1. Remove the Pulsar and Generate an Image}

In order to remove the pulsar from each of the 22 central $121 \times 121$ pixel $(6 ! .05 \times 6$ !"05) HST images, we first 

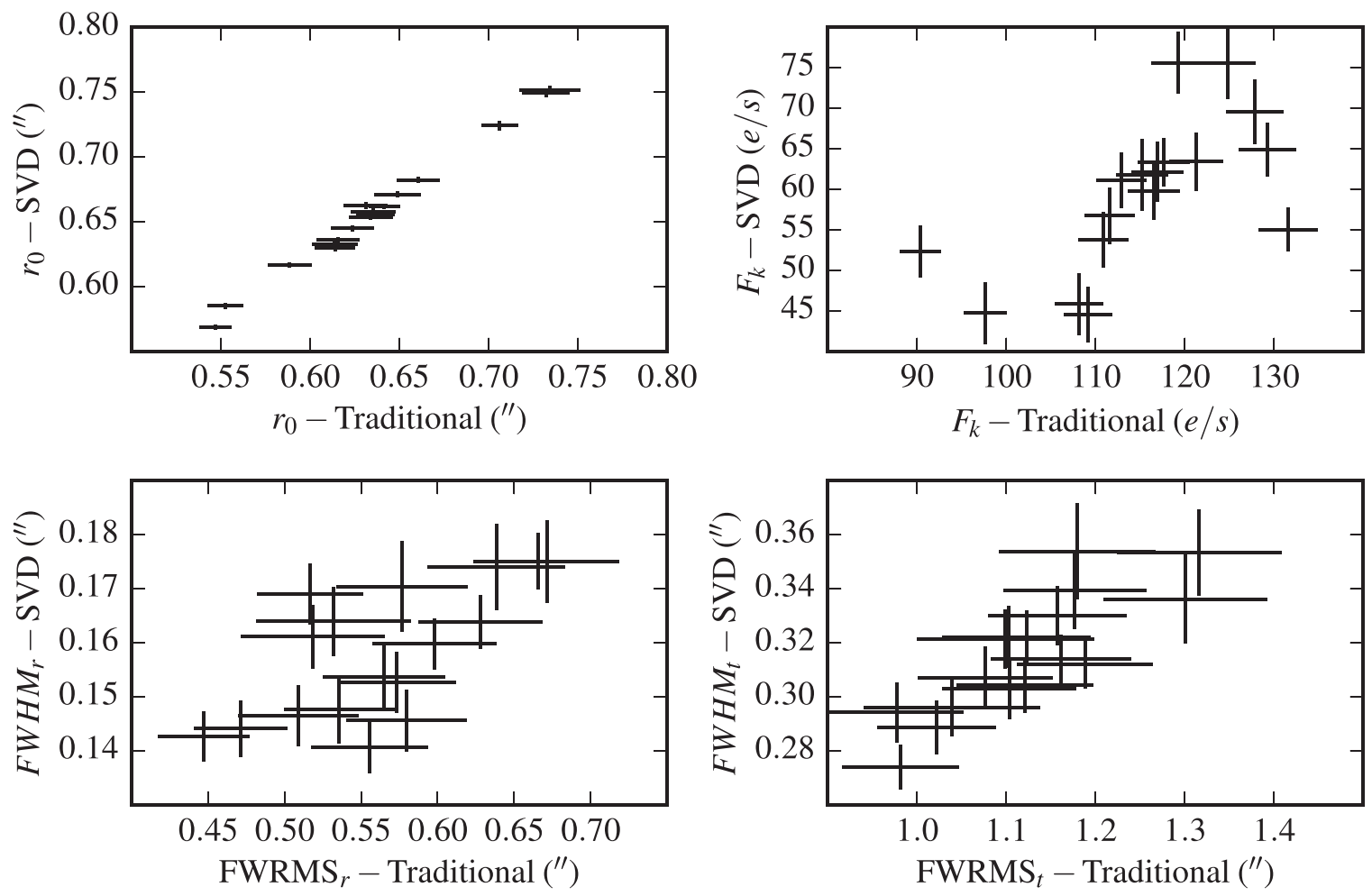

Figure 15. Comparison of HST-SVD and HST-traditional measured properties of the inner knot.

determine the PSF of the HST/ACS using $121 \times 121$ pixel images around each of the 19 isolated stars in the field. In doing this, we account for the fact that some observations were at a roll angle $\sim 180^{\circ}$ opposite that of the others. After subtracting a fitted linear gradient from the image of each (Figure 12) of the 19 stars and for each of the 22 observations, we register the resulting $418=19 \times 22$ star images and use SVD to generate a linear basis (lowest term shown in Figure 12) describing the PSF. The model pulsar image (Figure 12) uses the first 72 components of the SVD basis. After subtracting a linear gradient from each of the 22 central (pulsar) images (Figure 13), we fit each using the PSF basis to generate 22 residual (pulsar removed) images (also Figure 13), each now dominated by the inner knot.

\section{A.2.2.2. Characterize Inner-knot Properties}

The measured extrinsic properties of the inner knot are the projected radial separation $r_{0}$ and the polar angle $\psi_{0}$ of the peak surface brightness with respect to the pulsar. To facilitate characterization of the intrinsic properties of the inner knot, we map each inner knot sub-image (Figure 13) onto a $\psi-r$ grid (Figure 14). For an initial model of the surface brightness (intensity) distribution of the inner knot, we use a simple bivariate normal distribution: $S(r, \psi)=S_{\mathrm{k}} \exp \left(-\frac{1}{2}\left(\left(r-r_{0}\right)^{2} / \sigma_{r}^{2}+\left(\psi-\psi_{0}\right)^{2} / \sigma_{\psi}^{2}\right)\right)$.

The major intrinsic properties of the inner knot are its radial dispersion $\sigma_{r} \quad\left(\mathrm{FWHM}_{r}=2.35 \sigma_{r}\right.$ for a Gaussian profile), azimuthal dispersion $\sigma_{\psi}$ (or tangential dispersion $\sigma_{t}=r_{0} \sigma_{\psi}$ ), and peak intensity (surface brightness) $S_{\mathrm{k}}$. Integrating the intensity over a solid angle gives the flux $F_{\mathrm{k}}=2 \pi \sigma_{r} \sigma_{t} S_{\mathrm{k}}$. In fitting this model to the $\psi-r$ image of the inner knot, we include a constant-surface-brightness background $S_{\mathrm{b}}$ as a model parameter.
While this simple model provides adequate estimates to characterize the primary properties of the knot (Table 6), as a check we also introduced a somewhat more complicated model to deal with minor asymmetries in the $\psi-r$ plane. In particular, we took the radial location and radial width to be weak functions of the azimuthal angle, which we expand as a Taylor series to second order: $r \approx r(\psi) \approx r_{0}+r_{0}^{\prime}\left(\psi-\psi_{0}\right)+\frac{1}{2} r_{0}^{\prime \prime}\left(\psi-\psi_{0}\right)^{2}$ and $\quad \sigma_{r} \approx \sigma_{r}(\psi) \approx \sigma_{r_{0}}+\sigma_{r_{0}}^{\prime}\left(\psi-\psi_{0}\right)+\frac{1}{2} \sigma_{r_{0}}^{\prime \prime}\left(\psi-\psi_{0}\right)^{2}$. However, the derivatives proved not to significantly alter the results.

\section{A.2.2.3. Comparison Between Methods}

The comparison between methods and the reasons for somewhat emphasizing the SVD-based results considers the various measured parameters individually. Figure 15 compares measurements of similar variables between the two methods. From the figure we see that with the possible exception of the knot flux, both methods yield measurements that track each other apart from a scale factor. This leads to the result that the pulsar-knot separation determines all of the intrinsic properties of the knot.

Differences for $r_{0}$ are quite small, whereas the knot flux and size estimates differ by factors of $\approx 2$. We would expect differences in the size estimates, as the two analyses are using different measures of knot size- the first, FWRMS; the second, FWHM - that are not simply related for a non-Gaussian profile. That the fluxes differ is perhaps not so surprising, as its measurement is sensitive to the method of background subtraction and the impact of emission in the wings of the inner knot's profile. A significant difference between the two methods is the smaller uncertainty in $r_{0}$ afforded by the SVD approach, which removes those SVD components that represent noise, thus enhancing signal to noise. 


\section{A.3. Chandra}

Figure 6 shows the summed image at pulse minimum from the six "flaring" observations (ObsIDs 14684-16247 in Table 3). Prior to the binning used to produce the figure, we extract the counts in the three regions shown. It is difficult, if not impossible, to make use of the absolute number of counts as the different instrument configurations are all uncalibrated. However, we can make use of the relative numbers of counts from the different regions. Thus, the central region serves as an indicator of the number of pulsar counts, which can then be compared with the other observations to derive physical fluxes (assuming of course that the phase-averaged pulsar flux has not varied). The region to the southeast provides the upper limit for the flux in the knot and we use the region to the northwest to estimate the background, comprised mainly of that due to the wings of the pulsar PSF.

The alert reader will notice a slight excess of counts roughly 0 ". 6 to the southwest of the pulsar. This feature is seen both in the phase-averaged pulse and in the data from ObsID 11245. (The asymmetric effect of the blade inserted for ObsID 9765 does not allow these data to be used in making this comparison.) Therefore, we conclude that this feature is part of the pulsar PSF. A more subtle effect is also present as there is a slight asymmetry in the PSF that causes the region we associate with the inner knot to be somewhat brighter than the background during observations taken in the fall. In the spring, when the spacecraft has (naturally) been set at a roll angle that differs by $\approx 180^{\circ}$ from that in the fall, the background region is brighter than that we associate with the location of the inner knot. This effect is mainly seen in the phase-averaged data, and by comparing data from the ObsID 9765 (spring) and ObsID 11245 (fall) observations. Both effects described in this paragraph are relatively minor and are mainly due to the fact that we are working slightly below the spatial resolution of Chandra; thus, these effects have been ignored in setting the upper limits in Table 8.

\section{REFERENCES}

Aliu, E., Archambault, S., Aune, T., et al. 2014, ApJL, 781, LL11 Arons, J. 2012, SSRv, 173, 341

Baty, H., Petri, J., \& Zenitani, S. 2013, MNRAS, 436, L20
Beckwith, S. V. W., Stiavelli, M., Koekemoer, A. M., et al. 2006, AJ, 132, 1729

Bertin, E., \& Arnouts, S. 1996, A\&AS, 117, 393

Bogovalov, S. V. 1999, A\&A, 349, 1017

Buehler, R., Scargle, J. D., Blandford, R. D., et al. 2012, ApJ, 749, 26

Bühler, R., \& Blandford, R. 2014, RPPh, 77, 066901

Buson, S., Buehler, R., \& Hays, E. 2013, ATel, 5485, 1

Bykov, A. M., Pavlov, G. G., Artemyev, A. V., \& Uvarov, Y. A. 2012, MNRAS, 421, L67

Camus, N. F., Komissarov, S. S., Bucciantini, N., \& Hughes, P. A. 2009, MNRAS, 400, 1241

Cerutti, B., Uzdensky, D. A., \& Begelman, M. C. 2012, ApJ, 746, 148

Cerutti, B., Werner, G. R., Uzdensky, D. A., \& Begelman, M. C. 2013, ApJ, 770,147

Clausen-Brown, E., \& Lyutikov, M. 2012, MNRAS, 426, 1374

Fitzpatrick, E. L. 1999, PASP, 111, 63

Fruchter, A. S., \& Hook, R. N. 2002, PASP, 114, 144

Ghez, A. M., Salim, S., Weinberg, N. N., et al. 2008, ApJ, 689, 1044

H. E. S. S. Collaboration Abramowski, A., Aharonian, F., et al. 2014, A\&A, 562, A145

Hester, J. J. 2008, ARA\&A, 46, 127

Hester, J. J., Scowen, P. A., Sankrit, R., et al. 1995, ApJ, 448, 240

Komissarov, S. S., \& Lyubarsky, Y. E. 2003, MNRAS, 344, L93

Komissarov, S. S., \& Lyutikov, M. 2011, MNRAS, 414, 2017

Kron, R. G. 1980, ApJS, 43, 305

Lyubarsky, Y. E. 2012, MNRAS, 427, 1497

Lyutikov, M., Balsara, D., \& Matthews, C. 2012, MNRAS, 422, 3118

Lyutikov, M., Pariev, V. I., \& Blandford, R. D. 2003, ApJ, 597, 998

Mayer, M., Buehler, R., Hays, E., et al. 2013, ApJL, 775, L37

Melatos, A., Scheltus, D., Whiting, M. T., et al. 2005, ApJ, 633, 931

Moran, P., Shearer, A., Mignani, R. P., et al. 2013, MNRAS, 433, 2564

Ng, C.-Y., \& Romani, R. W. 2008, ApJ, 673, 411

Ojha, R., Hays, E., Buehler, R., \& Dutka, M. 2013, ATel, 4855, 1

Porth, O., Komissarov, S. S., \& Keppens, R. 2014, MNRAS, 438, 278

Rees, M. J., \& Gunn, J. E. 1974, MNRAS, 167, 1

Sandberg, A., \& Sollerman, J. 2009, A\&A, 504, 525

Sollerman, J. 2003, A\&A, 406, 639

Sollerman, J., Lundqvist, P., Lindler, D., et al. 2000, ApJ, 537, 861

Striani, E., Tavani, M., Piano, G., et al. 2011, ApJL, 741, L5

Sturrock, P., \& Aschwanden, M. J. 2012, ApJL, 751, L32

Tavani, M., Bulgarelli, A., Vittorini, V., et al. 2011, Sci, 331, 736

Tchekhovskoy, A., Philippov, A., \& Spitkovsky, A. 2015, arXiv:1503.01467

Tchekhovskoy, A., Spitkovsky, A., \& Li, J. G. 2013, MNRAS, 435, L1

Tennant, A. F., Becker, W., Juda, M., et al. 2001, ApJL, 554, L173

Teraki, Y., \& Takahara, F. 2013, ApJ, 763, 131

Tziamtzis, A., Lundqvist, P., \& Djupvik, A. A. 2009, A\&A, 508, 221

Uzdensky, D. A., Cerutti, B., \& Begelman, M. C. 2011, ApJL, 737, L40

Weisskopf, M. C., Tennant, A. F., Arons, J., et al. 2013, ApJ, 765, 56

Wizinowich, P. L., Le Mignant, D., Bouchez, A. H., et al. 2006, PASP, 118,297

Yelda, S., Lu, J. R., Ghez, A. M., et al. 2010, ApJ, 725, 331

Yuan, Q., Yin, P.-F., Wu, X.-F., et al. 2011, ApJL, 730, L15 